\title{
Active Disturbance Rejection Station-Keeping Control of Unstable Orbits around Collinear Libration Points
}

\author{
Min Zhu, ${ }^{1}$ Hamid Reza Karimi, ${ }^{2}$ Hui Zhang, ${ }^{1}$ Qing Gao, ${ }^{1,3}$ and Yong Wang ${ }^{1}$ \\ ${ }^{1}$ Department of Automation, University of Science and Technology of China, Hefei 230027, China \\ ${ }^{2}$ Department of Engineering, Faculty of Engineering and Science, University of Agder, 4876 Grimstad, Norway \\ ${ }^{3}$ School of Engineering and Information Technology, University of New South Wales at the Australian Defence Force Academy, \\ Canberra, ACT 2600, Australia
}

Correspondence should be addressed to Yong Wang; yongwang@ustc.edu.cn

Received 15 January 2014; Accepted 13 March 2014; Published 10 April 2014

Academic Editor: Shen Yin

Copyright (C) 2014 Min Zhu et al. This is an open access article distributed under the Creative Commons Attribution License, which permits unrestricted use, distribution, and reproduction in any medium, provided the original work is properly cited.

\begin{abstract}
An active disturbance rejection station-keeping control scheme is derived and analyzed for station-keeping missions of spacecraft along a class of unstable periodic orbits near collinear libration points of the Sun-Earth system. It is an error driven, rather than model-based control law, essentially accounting for the independence of model accuracy and linearization. An extended state observer is designed to estimate the states in real time by setting an extended state, that is, the sum of unmodeled dynamic and external disturbance. This total disturbance is compensated by a nonlinear state error feedback controller based on the extended state observer. A nonlinear tracking differentiator is designed to obtain the velocity of the spacecraft since only position signals are available. In addition, the system contradiction between rapid response and overshoot can be effectively solved via arranging the transient process in tracking differentiator. Simulation results illustrate that the proposed method is adequate for station-keeping of unstable Halo orbits in the presence of system uncertainties, initial injection errors, solar radiation pressure, and perturbations of the eccentric nature of the Earth's orbit. It is also shown that the closed-loop control system performance is improved significantly using our method comparing with the general LQR method.
\end{abstract}

\section{Introduction}

In recent years there has been an increasing interest in libration points missions. The libration points, which are normally called equilibrium points or Lagrangian points, correspond to regions in space where the centrifugal forces and the gravitational forces from the Sun and the Earth cancel each other. The existences of periodic orbits and quasi-periodic orbits in the vicinity of collinear libration points have been proved and analyzed rigorously in celestial mechanics [1, 2]. These orbits offer potentially valuable opportunities for investigations concerning solar and heliospheric effects on planetary environment and highly precise visible light telescopes.

However, these libration point orbits are inherently unstable but controllable. Thus, additional control force is needed for a spacecraft to remain close to the nominal orbit. The challenges of station-keeping control emerge from highly accuracy, low computation burden, and minimal fuel cost control requirements under the condition of spacecraft dynamic uncertainties, unmodeled perturbations, and initial orbit injection errors [3-6]. Hence, station-keeping control for libration point orbit missions is of virtual importance but with a great deal of difficulties.

The study of station-keeping control on libration point orbits has become a popular research topic ever since the problem was firstly proposed. A vast majority of the stationkeeping control methods are designed based on LTI model via local linearization at the libration points due to the high nonlinearity of the dynamic equation of libration point orbit. The nonlinear dynamic equation is obtained utilizing a Clohessy-Wiltshire- (CW-) like reference frame, which is 
widely used for Earth-centered spacecraft dynamic analysis $[7,8]$. Breakwell et al. $[9,10]$ firstly introduced classical optimal control strategies for Halo orbit missions. Erickson and Glass [11] specially analyzed the ISEE-3 mission to make this approach into implementation. Cielaszyk and Wie [12] employed a new LQR control method combined with a disturbance accommodating controller for Lissajous and Halo orbits maintenance based on LTI model. Di Giamberardino and Monaco [13] designed a nonlinear controller based on LTI model to solve the problem of tracking Halo orbit about the $L_{2}$ point. However, the LTI model includes only 1st, 2nd, or 3rd order term of the gravitational force and is only effective in the neighborhood of the libration point. As a matter of fact, the higher the amplitude of the libration point orbit, the greater the influence of nonlinear factors.

To improve the modeling accuracy, LTV method is employed instead of the LTI approach. Gurfil and Kasdin [14] expended the LTI model to LTV model and designed a timevarying controller for formation flying in the Sun-Earth system. Barcolona Group proposed the so-called Floquet Mode Control [15-17], which employs nonlinear techniques to compute the invariant manifolds and determine the discrete impulsive maneuvers to cancel deviations along the unstable component based on LTV model. Howell and Pernicka [18] developed a target-point approach to maintain the spacecraft within some torus about the nominal Halo orbit. Furthermore, several other control strategies are developed to overcome the disadvantage of searching for an optimal controller for both Floquet Mode approach and target-point approach. Kulkarni et al. [3] extended the traditional $H_{\infty}$ framework to periodic discrete LTV systems for stabilization of spacecraft flight in Halo orbits. Wang et al. [6] presented a nonlinear controller based on polynomial eigenstructure assignment of LTV model for the control of Sun-Earth $L_{2}$ point stationkeeping and formation flying, without considering the system uncertainties.

Besides the LTI and LTV model-based method, existing works also involve methodologies directly developed from original nonlinear dynamical equation of motion. Rahmani et al. [19] solved the problem of Halo orbit control using the optimal control theory and the variation of the extreme technique. Xin et al. [4] used a suboptimal control technique (the $\theta-D$ technique) to complete the mission of multiple spacecraft formation flying in deep space about the $L_{2}$ point. Marchand and Howell [20] employed the feedback linearization approach for formation flight in the vicinity of libration points. Gurfil et al. [21] presented a novel nonlinear adaptive neural control methodology for deep-space formation flying. Bai and Junkins [22] proposed a modified Chebyshev-Picard interation method for station-keeping of $L_{2}$ Halo orbits of Earth-Moon system. These nonlinear control methods have improved the control performance. However, the robustness under nonlinear system uncertainties and the request for low computation burden are unreasonably neglected, which should be considered before implementation.

Consequently, this paper derives an active disturbance rejection station-keeping control method considering mainly the following issues.
(1) Proposing an error driven, rather than model-based control law which takes into account system uncertainties, unmodeled disturbance, and orbit injection errors to achieve better robustness.

(2) Considering output feedback from practical viewpoint rather than full information, since only position signals of spacecraft can be measured.

(3) Better station-keeping performance as well as simpler computation burden.

A new nonlinear station-keeping control law based on active disturbance rejection control (ADRC) method, which refers to the so-called active disturbance rejection stationkeeping control (ADRSC) method in this paper, is proposed and analyzed. ADRC was firstly proposed by Han $[23,24]$, and Sun [25] and has been successfully applied in various industrial processes such as vehicle flight control, ship tracking control, robot control, and power plant control.

The remainder of this paper is organized as follows. Section 2 presents the equations of motion and periodic reference orbits about the Sun-Earth libration point. Section 3 presents an introduction to the theory of ADRC synthesis. Section 4 presents an ADRSC method for nonlinear stationkeeping of periodic orbits around collinear libration point. Section 5 carries out simulation results to validate the effectiveness of the new method. Finally, Section 6 concludes the paper.

\section{Equation of Motion}

In this section, the dynamic models of spacecraft based on the circular restricted three-body problem (CR3BP) are established. Additionally, the relative Halo reference orbits derived from LP map method are presented.

2.1. Dynamics of CR3BP. The CR3BP is one of the most common nonlinear models which investigate the relative motion around the libration points. As is shown in Figure 1, the infinitesimal mass $m$ of spacecraft compared to the mass $m_{1}$ of the Sun and the mass $m_{2}$ of the Earth is assumed. It is further assumed that the two primary bodies rotate about their barycenter in a circular orbit under a constant angular velocity $w$. A rotating coordinate system $(O, X, Y, Z)$ is defined with the origin set at the barycenter of the SunEarth system. The $X$-axis is directed from the Sun towards the Earth. The $Z$-axis is perpendicular to the plane of rotation and is positive when pointing upward. The $Y$-axis completes the set to yield a right-hand reference frame. Normalization is performed by defining the distance $R$ between the Sun and the Earth as the unit AU of length, the time of $1 / w$ as the unit of time TU, and the total mass of the Sun and the Earth as the unit of mass. Normalization is obtained by setting $\mu=m_{2} /\left(m_{1}+m_{2}\right), m_{2}=\mu$, and $m_{1}=1-\mu$, thus, $m_{1}$ is 


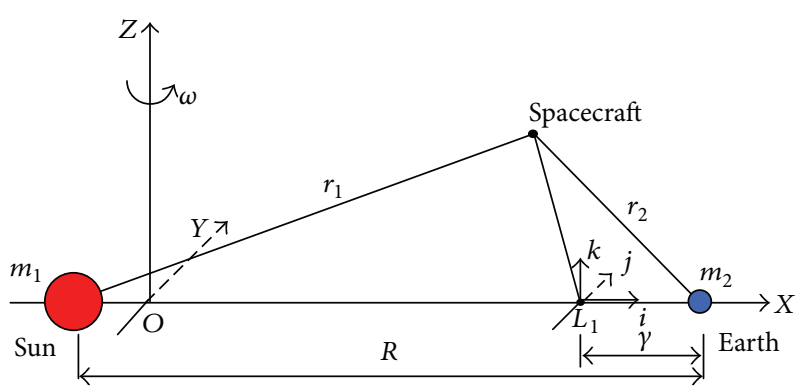

FIGURE 1: Restricted three-body problem.

located at $(-\mu, 0,0)$ and $m_{2}$ is located at $(1-\mu, 0,0)$. The wellknown equations of motion for CR3BP can be written in the dimensionless form [1]

$$
\begin{gathered}
\ddot{X}=2 \dot{Y}+X-\frac{(1-\mu)(X+\mu)}{r_{1}^{3}}-\frac{\mu(X-(1-\mu))}{r_{2}^{3}}, \\
\ddot{Y}=-2 \dot{X}+Y-\frac{(1-\mu) Y}{r_{1}^{3}}-\frac{\mu Y}{r_{2}^{3}}, \\
\ddot{Z}=-\frac{(1-\mu) Z}{r_{1}^{3}}-\frac{\mu Z}{r_{2}^{3}}
\end{gathered}
$$

where the dot represents time derivative in the rotating frame, and the distance $r_{1}=\sqrt{(X+\mu)^{2}+Y^{2}+Z^{2}}$ and $r_{2}=$ $\sqrt{(X-(1-\mu))^{2}+Y^{2}+Z^{2}}$.

2.2. Periodic Reference Orbits. We can find libration points of the Sun-Earth system, denoted by $L_{1}-L_{5}$, by setting all derivatives in (1) to zero. For the Sun-Earth system, $L_{1}$ is located at $X=0.989986055$. It has been proved that the three collinear points, $L_{1}, L_{2}$, and $L_{3}$, are unstable but controllable [1]. And a family of periodic orbits and quasi-periodic orbits exist around the collinear points [2].

Periodic solutions of the nonlinear equations of motion can be constructed using the method of successive approximations in conjunction with a technique similar to the Lindstedt-Poincare method [26]. These periodic solutions are firstly presented by Richardson $[27,28]$ and have been widely quoted and used. To obtain the periodic solutions around $L_{1}$, a new rotating coordinate system $\left(L_{1}, i, j, k\right)$ is defined with the origin set at $L_{1}$ and the distance $\gamma$ between the Earth and $L_{1}$ as the new unit of length, which is also shown in Figure 1. The relationship between the $(O, X, Y, Z)$ reference frame and the $\left(L_{1}, i, j, k\right)$ reference frame is as follows:

$$
i=\frac{X-(1-\mu-\gamma)}{\gamma}, \quad j=\frac{Y}{\gamma}, \quad k=\frac{Z}{\gamma},
$$

where $\gamma=0.010010905$ [27]. by

The equations of the Halo orbit to the third order are given

$$
\begin{aligned}
x_{r}(t)= & a_{21} A_{x}^{2}+a_{22} A_{z}^{2}-A_{x} \cos (\lambda t) \\
& +\left(a_{23} A_{x}^{2}-a_{24} A_{z}^{2}\right) \cos (2 \lambda t) \\
& +\left(a_{31} A^{3}-a_{32} A_{x} A_{z}^{2}\right) \cos (3 \lambda t), \\
y_{r}(t)= & \left(A_{y}+b_{33} A_{x}^{3}+\left(b_{34}-b_{35}\right) A_{x} A_{z}^{2}\right) \sin (\lambda t) \\
& +\left(b_{21} A_{x}^{2}-b_{22} A_{z}^{2}\right) \sin (2 \lambda t) \\
& +\left(b_{31} A_{x}^{3}-b_{32} A_{x} A_{z}^{2}\right) \sin (3 \lambda t), \\
z_{r}(t)= & -3 d_{21} A_{x} A_{z}+A_{z} \cos (\lambda t) \\
& +d_{21} A_{x} A_{z} \cos (2 \lambda t) \\
& +\left(d_{32} A_{z} A_{x}^{2}-d_{31} A_{z}^{3}\right) \cos (3 \lambda t),
\end{aligned}
$$

where $x_{r}(t), y_{r}(t)$ and $z_{r}(t)$ are the coordinates along $i, j$, and $k$ axes. The Halo orbit designed for ISEE-3 mission [27] is selected as one of the target station-keeping orbits. The other reference orbit is selected with higher vertical amplitude. The values of the various constants in (2) of both Halo orbits are given in Table 1.

\section{Introduction of ADRC}

Due to complexity of modern systems, more attention has been paid on data-driven control scheme recently $[29,30]$. The ADRC is a typical data-driven control. It inherits from PID, using the error driven, rather than model-based control law to eliminate errors.

Han's ADRC consists of three parts, a nonlinear tracking differentiator (TD) [31], which is used to arrange the ideal transient process of the system, an extended state observer (ESO) [32], which could estimate the disturbance from the system output and then the ADRC compensates the disturbances according to estimated values, and a nonlinear state error feedback (NLSEF) [24], which is used to get the control input of the system.

Consider the following system:

$$
\begin{gathered}
x_{1}=y, \\
\dot{x}_{1}=x_{2}, \\
\dot{x}_{2}=f\left(x_{1}, x_{2}, w(t), t\right)+b u,
\end{gathered}
$$

where $y$ is the output variable, $u$ is the control input, $b$ is magnification factor, and $\omega(t)$ is the external disturbance; $f\left(x_{1}, x_{2}, w(t), t\right)$ includes three parts: modeling dynamics, uncertain dynamics, and disturbance. The ADRC approach makes an effort to compensate for the unknown dynamics and the external disturbances in real time without an explicit mathematical expression. 
TABLE 1: The values of the various constants in (2) for ISEE-3 $(Z=110,000 \mathrm{~km})$ orbit and Halo orbit $(Z=800,000 \mathrm{~km})$ near $L_{1}$ libration point of the Sun-Earth system. For ISEE-3 $(Z=110,000 \mathrm{~km})$ orbit and for Halo orbit $(Z=800,000 \mathrm{~km})$.

\begin{tabular}{lccccc}
\hline Parameters & Value & Parameters & Value & Parameters & Value \\
\hline$\lambda$ & 2.08645 & $k$ & 3.22927 & $a_{21}$ & 2.09270 \\
$a_{22}$ & $2.48298 \times 10^{-1}$ & $a_{23}$ & $-9.05965 \times 10^{-1}$ & $a_{24}$ & $-1.04464 \times 10^{-1}$ \\
$a_{31}$ & $7.93820 \times 10^{-1}$ & $a_{32}$ & $8.26854 \times 10^{-2}$ & $b_{21}$ & $-4.92446 \times 10^{-1}$ \\
$b_{22}$ & $6.07465 \times 10^{-2}$ & $b_{31}$ & $8.85701 \times 10^{-1}$ & $b_{32}$ & $2.30198 \times 10^{-2}$ \\
$b_{33}$ & -2.84508 & $b_{34}$ & -2.30206 & $b_{35}$ & -1.87037 \\
$d_{21}$ & $-3.46865 \times 10^{-1}$ & $d_{31}$ & $1.90439 \times 10^{-2}$ & $d_{32}$ & $3.98095 \times 10^{-1}$ \\
\hline
\end{tabular}

3.1. TD. The TD has the ability to track the given input reference signal with quick response and no overshoot by providing transition process for expected input $v$ and differential trajectory of set value, that is, $v_{1}$, and its differential $v_{2}$.

One feasible second-order TD can be designed as [31]

$$
\begin{gathered}
\dot{v}_{1}=v_{2}, \\
\dot{v}_{2}=\operatorname{fhan}\left(v_{1}-v(t), v_{2}, r, h_{0}\right),
\end{gathered}
$$

where $v(t)$ denotes the control objective, $r$ is speed factor and decides tracking speed, $h_{0}$ is filtering factor, and fhan $\left(v_{1}-\right.$ $\left.v(t), v_{2}, r, h_{0}\right)$ is as follows:

$$
\begin{gathered}
d=r h_{0}^{2}, \\
a_{0}=h_{0} v_{2}, \\
y=\left(v_{1}-v(t)\right)+a_{0}, \\
a_{1}=\sqrt{d(d+8|d|)}, \\
a_{2}=a_{0}+\frac{\operatorname{sign}(y)\left(a_{1}-d\right)}{2}, \\
s_{y}=\frac{\operatorname{sign}(y+d)-\operatorname{sign}(y-d)}{2}, \\
a=\left(a_{0}+y-a_{2}\right) s_{y}+a_{2}, \\
s_{a}=\frac{\operatorname{sign}(a+d)-\operatorname{sign}(a-d)}{2}, \\
\text { fhan }\left(v_{1}-v(t), v_{2}, r, h_{0}\right) \\
=-r\left(\frac{a}{d}-\operatorname{sign}(a)\right) s_{a}-r \operatorname{sign}(a) .
\end{gathered}
$$

3.2. ESO. ESO is used to estimate $f\left(x_{1}, x_{2}, w(t), t\right)$ in real time and to make adjustments at each sampling point in a digital controller. An augmented variable $x_{3}=f\left(x_{1}, x_{2}, w(t), t\right)$ is introduced in (4). Using $z_{1}, z_{2}$, and $z_{3}$ to estimate $x_{1}, x_{2}$, and $x_{3}$, respectively, a nonlinear observer is designed as [32]

$$
\begin{gathered}
e=z_{1}-y, \\
\dot{z}_{1}=z_{2}-\beta_{1} e, \\
\dot{z}_{2}=z_{3}-\beta_{2} \mathrm{fal}\left(e, \alpha_{1}, \delta\right)+b u, \\
\dot{z}_{3}=-\beta_{3} \mathrm{fal}\left(e, \alpha_{2}, \delta\right),
\end{gathered}
$$

where $\beta_{1}, \beta_{2}$, and $\beta_{3}$ are observer gains, $e$ is the error, and $\mathrm{fal}(x, \alpha, \delta)$ is as follows:

$$
\operatorname{fal}(x, \alpha, \delta)= \begin{cases}\frac{x}{\delta^{1-\alpha}}, & |x| \leq \delta \\ \operatorname{sign}(x)|x|^{\alpha}, & |x|>\delta .\end{cases}
$$

3.3. NLSEF. NLSEF generates control voltage $u$ for system by using the errors between the output of ESO and TD

$$
\begin{aligned}
& e_{1}=v_{1}-z_{1}, \\
& e_{2}=v_{2}-z_{2} .
\end{aligned}
$$

A nonlinear combination of errors signal can be constructed as [24]

$$
u_{0}=- \text { fhan }\left(e_{1}, c e_{2}, \alpha, h_{1}\right),
$$

where the nonlinear coefficient $\alpha$ is selected as $0<\alpha<1$, and $c$ is the proportional coefficients.

The controller is designed as

$$
u=u_{0}-\frac{z_{3}}{b} .
$$

\section{ADRSC for Station-Keeping}

As is mentioned in Section 2, the reference Halo orbit around collinear libration orbit is inherently unstable, and station-keeping control approach is designed under model uncertainties and disturbances. In this paper, we extend the ADRC for the ADRSC. The structure of ADRSC algorithm is presented in Figure 2. ADRSC will be proposed and analyzed in this section from the following three aspects: discrete TD, discrete ESO, and discrete NLSEF.

4.1. Discrete TD for the Tracking of the Reference Orbit and the Spacecraft Trajectory. Assuming that only position signals of spacecraft can be detected and used for feedback, there are two different kinds of discrete TDs designed for stationkeeping control.

4.1.1. TD I. It is designed for reference Halo orbit tracking

$$
\begin{gathered}
e=v_{i 1}(k)-v_{i}(k), \\
v_{i 1}(k+1)=v_{i 1}(k)+h \times v_{i 2}(k), \\
v_{i 2}(k+1)=v_{i 2}(k)+h \times \operatorname{fhan}\left(e, v_{i 2}(k), r_{i}, h_{i}\right),
\end{gathered}
$$




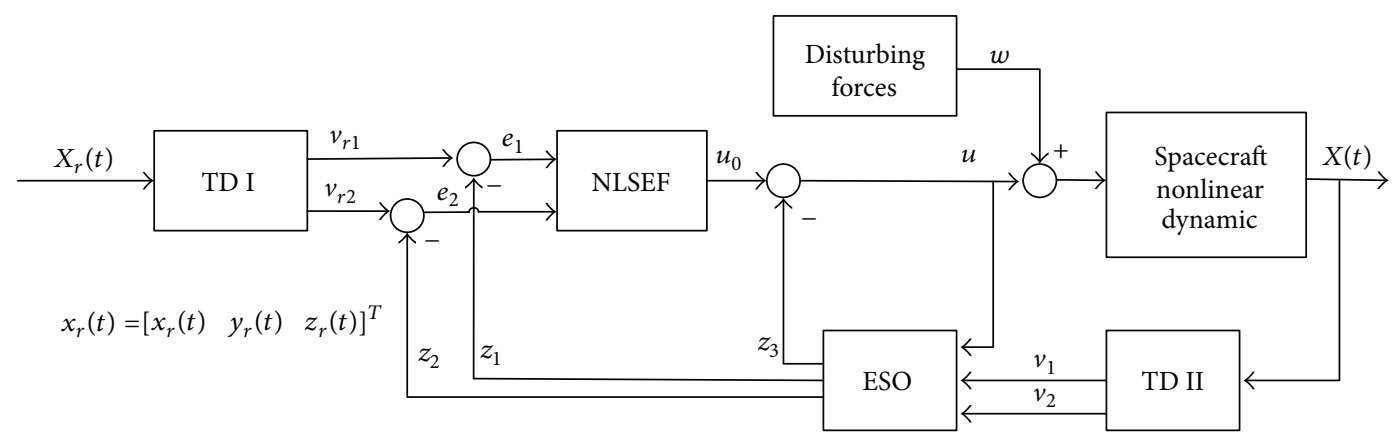

FIGURE 2: The structure of ADRSC algorithm.

where $i=1,2,3$ denote the $X, Y$, and $Z$ axes component, respectively, $v_{i}(k)$ denotes the reference orbit. TD I provides transition process for nominal orbit $v_{i}$ and differential trajectory of set position value, that is, $v_{i 1}$ and its differential $v_{i 2}$.

4.1.2. TD II. It is designed for flight position tracking and estimating of the velocity of the spacecraft

$$
\begin{gathered}
e=v_{i 1}(k)-v_{i}(k), \\
v_{i 1}(k+1)=v_{i 1}(k)+h \times v_{i 2}(k), \\
v_{i 2}(k+1)=v_{i 2}(k)+h \times \text { fhan }\left(e, v_{i 2}(k), r_{0 i}, h_{0 i}\right),
\end{gathered}
$$

where $i=1,2,3$ denote the $X, Y$, and $Z$ axes component, respectively. For instance, state vector $\left[\begin{array}{ll}v_{11} & v_{12}\end{array}\right]^{T}$ denotes the estimation of $\left[\begin{array}{ll}X & \dot{X}\end{array}\right]^{T}$ which is the position and velocity vector of spacecraft trajectory along $X$ axes. $v_{i}(k)$ denotes the flight trajectory of spacecraft. And $r_{0 i}$ is the tracking speed parameter and $h_{0 i}$ is the filter factor, which makes an effort of filtering. While the integration step is fixed, increasing the filtering factor will make the effort of filter better. The outputs of TD II, which are the spacecraft flight position and velocity, provide the inputs of ESO for feedback as described in Figure 2.

4.2. Discrete ESO for Estimating the Nonlinear Dynamics Model Uncertainties and Unmodeled Disturbance. The equations of station-keeping of Halo orbits using ADRSC can be generally defined as

$$
\begin{gathered}
\dot{x}_{i 1}=x_{i 2}, \\
\dot{x}_{i 2}=g_{i}(\mathbf{X}, \dot{\mathbf{X}})+w_{i}+u_{i}, \\
y=x_{i 1},
\end{gathered}
$$

where $i=1,2,3$ denote the $X, Y$, and $Z$ axes component, respectively. $\mathbf{X}=\left[\begin{array}{lll}x_{11} & x_{21} & x_{31}\end{array}\right]^{T}$ denotes the position vector of the spacecraft, $\mathbf{w}=\left[\begin{array}{lll}w_{1} & w_{2} & w_{3}\end{array}\right]^{T}$ denotes the disturbance vector, and $\mathbf{u}=\left[\begin{array}{lll}u_{1} & u_{2} & u_{3}\end{array}\right]^{T}$ denotes the vector of stationkeeping control input. $\mathbf{g}(\mathbf{X}, \dot{\mathbf{X}})=\left[\begin{array}{lll}g_{1} & g_{2} & g_{3}\end{array}\right]^{T}$ represents the uncertain dynamics vector, respectively. Note that (1) will be simulated as the real flight dynamic mode. Hence, robust performance will be presented under such formation of dynamic model uncertainties.

It is necessary to point out that a distinct improvement can be obtained using ADRSC for station-keeping since the dynamic model does not need to be expressively known which is different with the aforementioned studies. Define $\mathbf{F}(\mathbf{X}, \dot{\mathbf{X}})=\mathbf{g}(\mathbf{X}, \dot{\mathbf{X}})+\mathbf{w}$. In fact, in the context of feedback control, $\mathbf{F}(\mathbf{X}, \dot{\mathbf{X}})$ is something to be overcome by the control signal, and it is therefore denoted as the "total disturbance." At this point, $\mathbf{F}(\mathbf{X}, \dot{\mathbf{X}})$ is extended as an additional state variable; that is,

$$
x_{i 3}=F_{i}(\mathbf{X}, \dot{\mathbf{X}})
$$

where $F_{1}, F_{2}$, and $F_{3}$ denote the corresponding axis components of $\mathbf{F}(\mathbf{X}, \dot{\mathbf{X}})$, respectively, and let $\dot{\mathbf{F}}(\mathbf{X}, \dot{\mathbf{X}})=\mathbf{a}(\mathbf{X}, \dot{\mathbf{X}})$, where $\mathbf{a}(\mathbf{X}, \dot{\mathbf{X}})$ is unknown.

One can rewrite (14) as follows:

$$
\begin{gathered}
\dot{x}_{i 1}=x_{i 2}, \\
\dot{x}_{i 2}=x_{i 3}+u_{i}, \\
\dot{x}_{i 3}=a_{i}, \\
y=x_{i 1},
\end{gathered}
$$

where $x_{i 1}, x_{i 2}$, and $x_{i 3}$ represent the position, velocity, and $F_{i}(\mathbf{X}, \dot{\mathbf{X}})$, respectively, $i=1,2,3$. Then, one can use the following discrete nonlinear observer $\left\{z_{i 1}, z_{i 2}, z_{i 3}\right\}$ to estimate state vector $\left\{x_{i 1}, x_{i 2}, x_{i 3}\right\}$ :

$$
\begin{gathered}
e_{i}=z_{i}-y_{i}, \\
z_{i 1}(k+1)=z_{i 1}(k)+h \times\left(z_{i 2}(k)-\beta_{i 1} e\right), \\
z_{i 2}(k+1) \\
=z_{i 2}(k)+h \times\left(f_{0 i}+z_{i 3}(k)-\beta_{i 2} \times \operatorname{fal}(e, 0.5, h)\right) \\
+u(i), \\
z_{i 3}(k+1)=z_{i 3}(k)-h \times \beta_{i 3} \times \text { fal }(e, 0.25, h) .
\end{gathered}
$$

4.3. Discrete NLSEF for Station-Keeping. Since the states, unmodeled dynamics, and disturbances have been estimated by ESO presented in Section 4.2, state errors between the 
output of ESO and TD I are combined for feedback control law, large errors corresponding to low gains and small errors corresponding to high gains

$$
u_{i}(k+1)=- \text { fhan }\left(e_{i 1}, c_{i} e_{i 2}, \alpha_{i}, h_{i}\right)-z_{i 3}(k),
$$

where $\left\{c_{i}, \alpha_{i}, h_{i}\right\}$ are the NLSEF parameters described in (10). $e_{i 1}$ and $e_{i 2}$ are the state errors described in (9). $z_{i 3}$ denotes unmodeled dynamics and disturbances $F_{i}(\mathbf{X}, \dot{\mathbf{X}})$ which is estimated from (17).

The parameter $h_{i}$ of NLSEF, which is also named filter factor of fhan, makes great contribution in NLSEF control performance. With the integration step fixed, $h_{i}$ must be selected properly. It is noted that larger errors corresponding to larger $h_{i}$ and smaller errors corresponding to smaller $h_{i}$ will make a better performance.

In this paper, a parameter self-turning approach is firstly proposed for $h_{i}$ selection as follows:

$$
h_{i}= \begin{cases}k \log _{10} \sqrt{\sum_{i=1}^{3} e_{i 1}^{2}}+h_{0}, & \sqrt{\sum_{i=1}^{3} e_{i 1}^{2}}>\xi \\ k \log _{10} \xi+h_{0}, & \sqrt{\sum_{i=1}^{3} e_{i 1}^{2}} \leq \xi,\end{cases}
$$

where the parameters $k, h_{0}$, and $\xi$ of self-turning $h_{i}$ can be easily chosen in practice, as will be presented in the simulation.

4.4. Disturbing Forces. In any actual mission, the perturbation factors and the injection errors, coupled with the inherent unstable nature of the Halo orbits around the collinear libration points, will cause a spacecraft to drift from the periodic reference orbit [33]. A robust performance in the presence of disturbance can be obtained by ADRSC, and the model of disturbance is not necessary for ADRSC as long as the disturbance is bounded. Physically speaking, deepspace disturbances are always bounded [21]. To illustrate the capability of ADRSC to reject unknown disturbances, here we introduce the perturbative forces due to the eccentric nature of the Earth's orbit and the solar radiation pressure (SRP) disturbance.

4.4.1. The Perturbative Forces due to the Eccentric Nature of the Earth's Orbit. The most important perturbative effects in the CR3BP are the eccentric nature of the Earth's orbit and the gravitational force of the moon $[3,28]$. In this paper, the largest perturbative force per unit mass $\mathbf{d}_{\mathrm{ENE}}$ of spacecraft due to the eccentric nature of the Earth's orbit is taken into account, which has been given approximately in [3]

$$
\left|\mathbf{d}_{\mathrm{ENE}}\right|=G M_{\mathrm{Earth}}\left|\frac{1}{r_{c}^{2}}-\frac{1}{r_{e}^{2}}\right|,
$$

where $r_{e}$ is the position vector of the Earth at the pericenter of the Earth's elliptical orbit from the libration point. $r_{c}$ is the position vector similar to a circular orbit.
4.4.2. SRP Disturbance. In the deep-space mission, SRP is another disturbance to account for. Here, we adopt a widely used model [34]. According to this model, the disturbance acceleration to SRP is

$$
\mathbf{d}_{\mathrm{SRP}}=-\frac{\beta}{\left\|\mathbf{r}_{1}\right\|^{2}}\left(\widehat{\mathbf{r}}_{1} \cdot \mathbf{n}\right)^{2} \mathbf{n},
$$

where $\beta$ is a parameter that depends on the coefficient of reflectivity, the area, and mass of the spacecraft, the solar flux, and the speed of light. $\widehat{\mathbf{r}}_{1}=\mathbf{r}_{1} /\left\|\mathbf{r}_{1}\right\|$, and $\mathbf{n}$ is the attitude vector of the spacecraft in the rotating frame. To simplify (21), we assume that $\mathbf{n}=\widehat{\mathbf{r}}_{1}$, which yields

$$
\mathbf{d}_{\mathrm{SRP}}=-\frac{\beta}{\left\|\mathbf{r}_{1}\right\|^{3}} \mathbf{r}_{1} \text {. }
$$

\section{Simulation Results and Analysis}

5.1. Simulation Scenario and Numerical Values. To compare the performance of ADRSC with linearization method, the simulation of an LQR controller [1] based on LTI system from local linearization around $L_{1}$ point of nonlinear system equation (1) is also carried out. In order to compare the performance of ADRSC between low and large amplitude orbits, we select two orbits for simulation, one orbit for the ISEE-3 mission with vertical displacement $110,000 \mathrm{~km}$ and the other of $800,000 \mathrm{~km}$.

The control forces for station-keeping are provided by an ionic engine with the maximum thrust $60 \mathrm{mN}$ which is currently commercially available [35]. The mass of the spacecraft is designed as $m=500 \mathrm{~kg}$.

The disturbing forces described in 4.4 are considered in the simulation. The eccentricity of Earth orbit $e=0.016675$. And the SRP parameter is calculated assuming that the solar flux is $F_{s}=1358 \mathrm{~W} / \mathrm{m}^{2}$, the speed of light is $c=3 \times 10^{8} \mathrm{~m} / \mathrm{s}$, the cross-sectional area is $S=3.5 \mathrm{~m}^{2}$, and the coefficient of reflectivity is $q=0.6$, which results in $\beta=S F_{s}(1+q) /(m c)=$ $5 \times 10^{-8} \mathrm{~m} / \mathrm{s}^{2}$ [21]. It is also assumed that the initial position injection errors along the $x, y$, and $z$ directions are $1000 \mathrm{~km}$ and the velocity injection errors are $1 \mathrm{~m} / \mathrm{s}$. The MATLAB Simulink software is used for simulation. The time step $h$ is set as $0.0001 \mathrm{TU}$ for better accuracy of the spacecraft. The simulation time is chosen as 1800 days, almost 10 periods of Halo motion about $L_{1}$ libration point.

In the simulation, considering the system is not full state feedback, the TD is designed to track the spacecraft flight position and obtain the velocity as well. Figure 3 illustrates the TD II tracking result of the spacecraft flight. In Figure 3, FP and FV denote the spacecraft flight position and velocity; TP and TV denote the TD tracking position and velocity, respectively. Figure 3 presents the accurately tracking of position signal and velocity signal along the $x, y$, and $z$ axes, which will be used for feedback control.

\subsection{ISEE-3 Halo Orbit $(Z=110,000 \mathrm{~km})$}

5.2.1. Related Parameters. In dimensionless coordinates the initial orbit parameters without injection errors of ISEE-3 are 

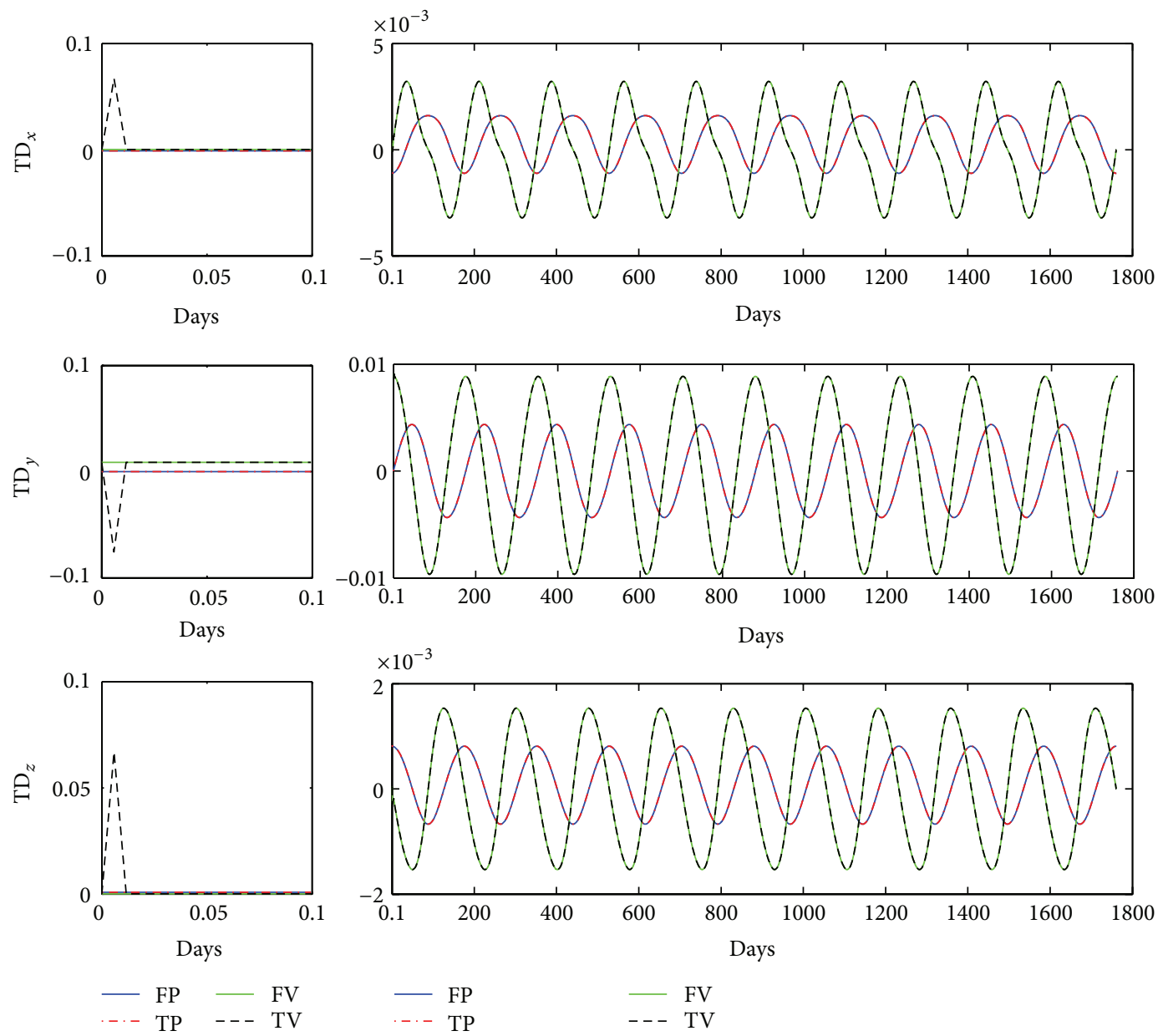

FIGURE 3: The TD tracking result.

TABLE 2: Initial orbits parameters of Halo missions without injection errors.

\begin{tabular}{lccccc}
\hline Mission orbits & $X_{0}$ & $Y_{0}$ & $Z_{0}$ & $\dot{X}_{0}$ & $\dot{Y}_{0}$ \\
\hline ISEE-3 $(110,000 \mathrm{~km})$ & 0.988872932669558 & 0 & $8.109335378305802 e-04$ & 0 & 0.008853797264729 \\
Halo $(800,000 \mathrm{~km})$ & 0.989390221855232 & 0 & 0.006248517078177 & 0 & 0.012547011257083 \\
\hline
\end{tabular}

given in Table 2 (in unit of AU for position and in unit of AU/TU for velocity). The parameters of ADRSC of ISEE-3 are given as follows: $r_{i}=1000, r_{0 i}=2000, \beta_{12}=\beta_{22}=300000$, $\beta_{11}=\beta_{21}=\beta_{13}=\beta_{23}=5000, \beta_{32}=100000, \beta_{31}=\beta_{33}=$ 2000; $c_{i}=0.5, \alpha_{i}=200, i=1,2,3, k=0.0005, h_{0}=-0.0004$, and $\xi=10$. The weight matrices of the LQR controller are selected as $Q=\operatorname{diag}\left\{3 \times 10^{8}, 3 \times 10^{8}, 3 \times 10^{8}, 3 \times 10^{6}, 3 \times\right.$ $\left.10^{6}, 3 \times 10^{6}\right\}, R=\mathbf{I}_{3}$.

5.2.2. Simulation Results. Figures 4 and 5 illustrate the station-keeping control results for ISEE-3 with orbit injection errors as well as disturbing forces, which show that preferable station-keeping accuracy can be obtained using ADRSC through $60 \mathrm{mN}$ engine. From Figures 5(a) and 5(b), the errors of relative position and relative velocity drop down very quickly and steadily to zero within 5 days compared with LQR controller 14 days in Figures 6(a) and 6(b). The position errors along $x, y$, and $z$ directions can be kept within $1 \mathrm{~km}$, which is very small compared with the orbit altitude of the ISEE-3 mission $(110,000 \mathrm{~km})$. Also, as shown in Figure 5(c) the control forces begin at $60 \mathrm{mN}$ to drive the relative position errors to zero, but quickly reduce to the steady progress in 5 days. Hence, these results illustrate the effectiveness of the ADRSC of unstable Halo orbits near collinear libration points.

In order to describe the results of ADRSC more accurately when the spacecraft moves steady on the Halo orbit, here 


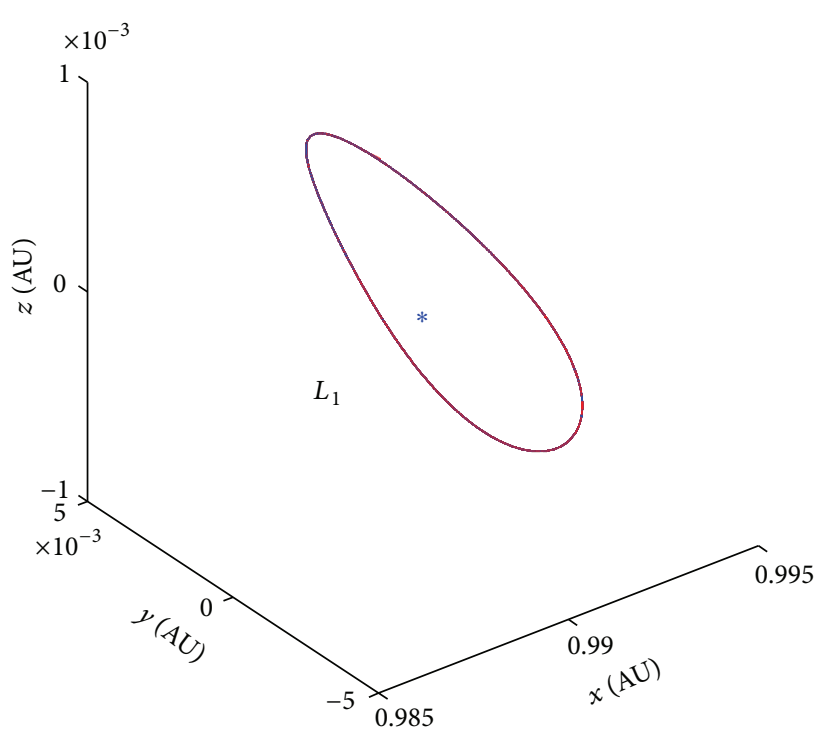

(a)

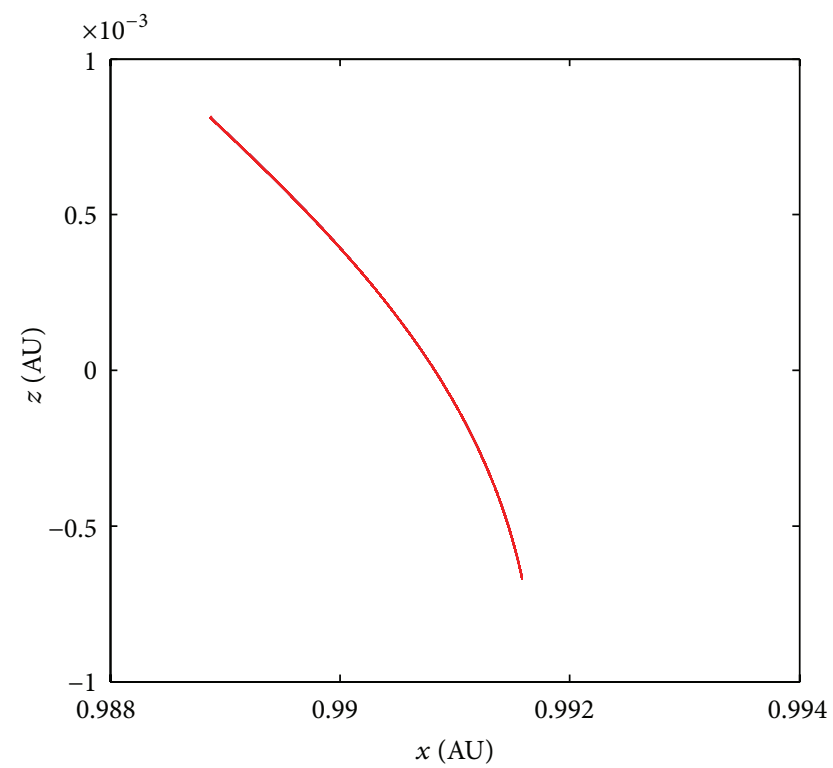

(c)

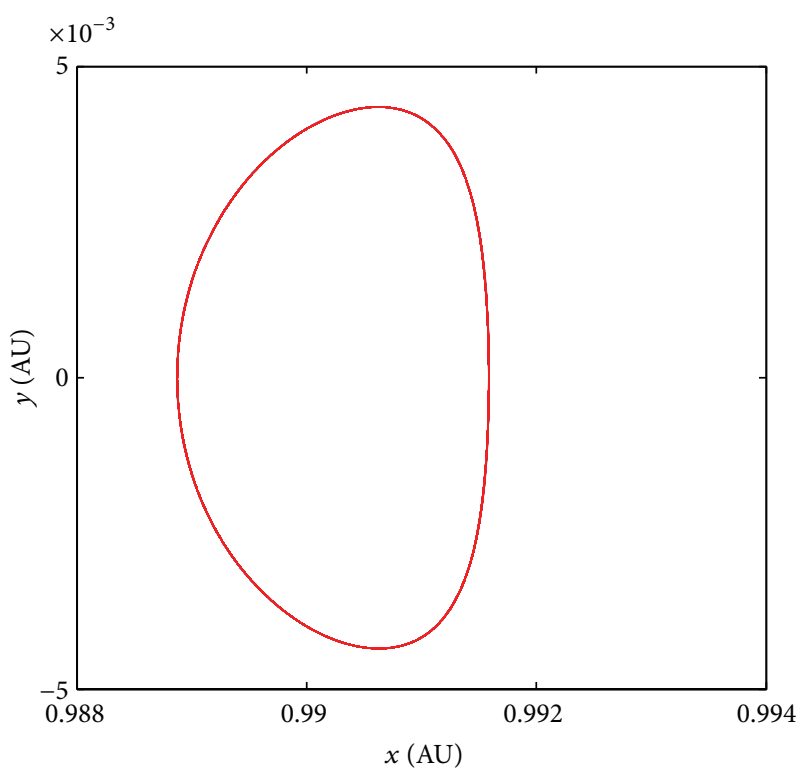

(b)

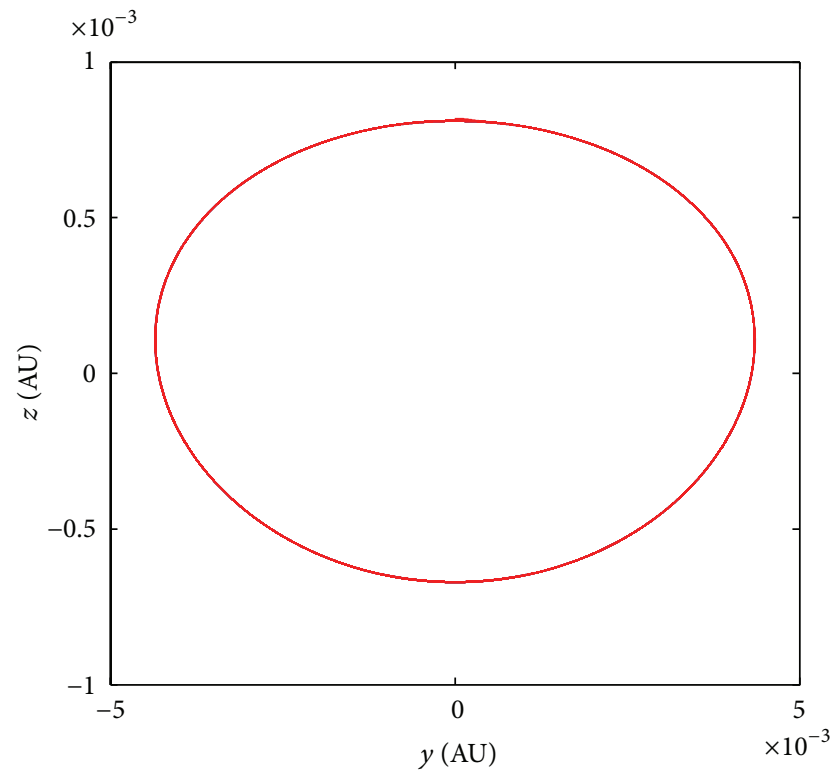

(d)

Figure 4: ADRSC of ISEE-3 $(Z=110,000 \mathrm{~km})$.

we introduce the popular evaluation parameters for stationkeeping $[3,12,33]$, the velocity increments $\Delta V_{x}, \Delta V_{y}$, and $\Delta V_{z}$ (in unit of $\mathrm{m} / \mathrm{s} / \mathrm{T}$ ), and the mean absolute value of the position errors $e_{x}, e_{y}$, and $e_{z}$ (in unit of $\mathrm{km}$ ) as follows [33]:

$$
\begin{gathered}
\Delta V_{i}=\frac{1}{T} \int_{0}^{T}\left|u_{i}\right| \mathrm{d} t \quad(i=x, y, z), \\
\Delta V=\sqrt{\Delta V_{x}^{2}+\Delta V_{y}^{2}+\Delta V_{z}^{2}}, \\
e_{i}=|\Delta i|_{\text {mean }} \quad(i=x, y, z), \quad e=\sqrt{e_{x}^{2}+e_{y}^{2}+e_{z}^{2}},
\end{gathered}
$$

where $T$ is the orbitalperiod.
The velocity increment and the mean absolute value of the position errors of ISEE-3 station-keeping with orbit injection errors and disturbance are given in Table 3 . It can be seen that the mean absolute position error is $0.389 \mathrm{~km}$, which illustrates the higher precision characteristic for the spacecraft station-keeping mission with ADRSC, comparing with that of LQR controller $2.965 \mathrm{~km}$ shown in Table 3. Meanwhile, the velocity increment of ADRSC under disturbing force and injection error is $36.567 \mathrm{~m} / \mathrm{s} / \mathrm{T}$ which is approximately equal to that of LQR controller $36.705 \mathrm{~m} / \mathrm{s} / \mathrm{T}$. Note that there is a trade-off between accuracy of the spacecraft to follow the Halo orbit and the thrust usage [3]. The earlier approaches, which use impulsive maneuvers, are designed 

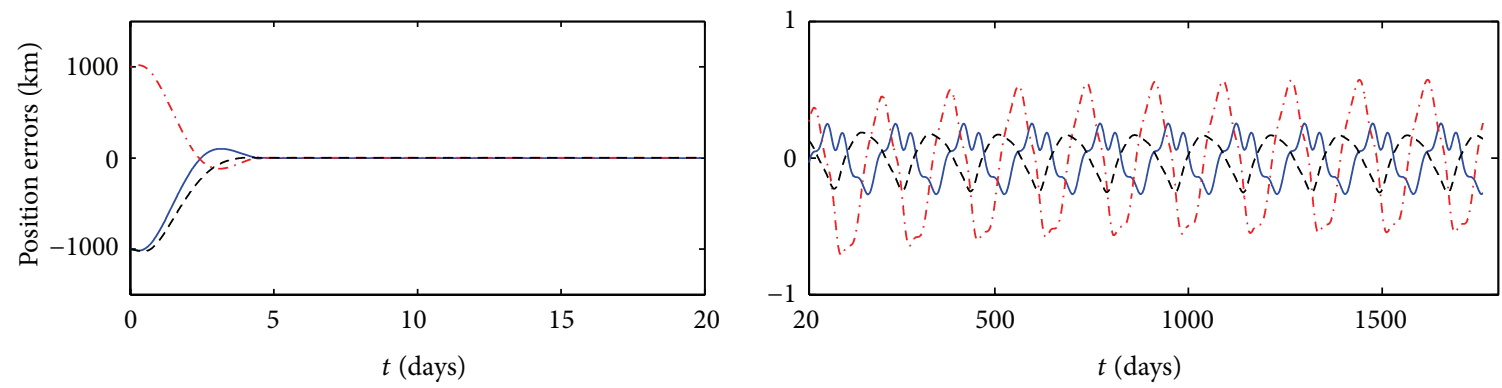

(a)
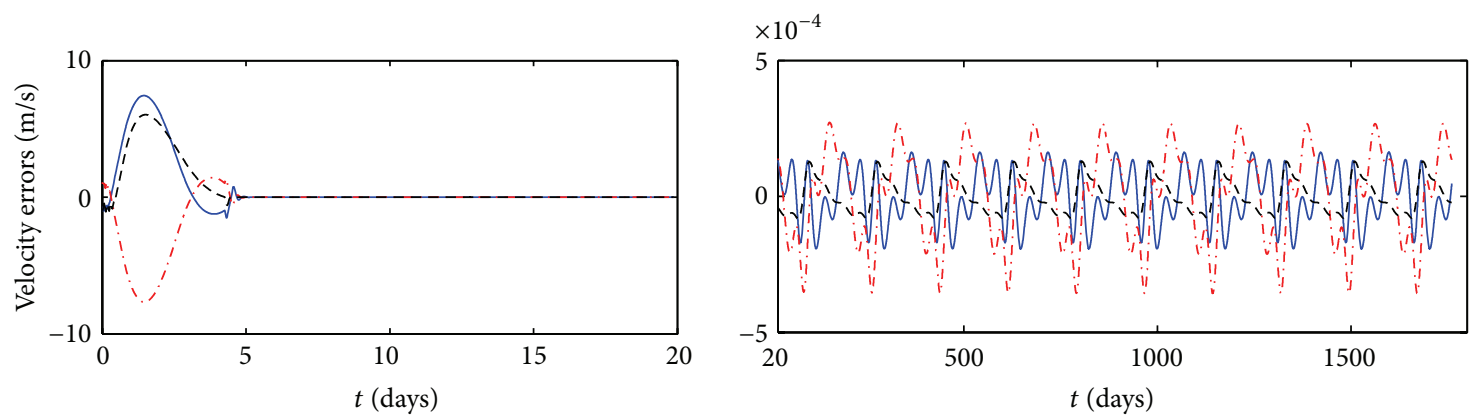

(b)
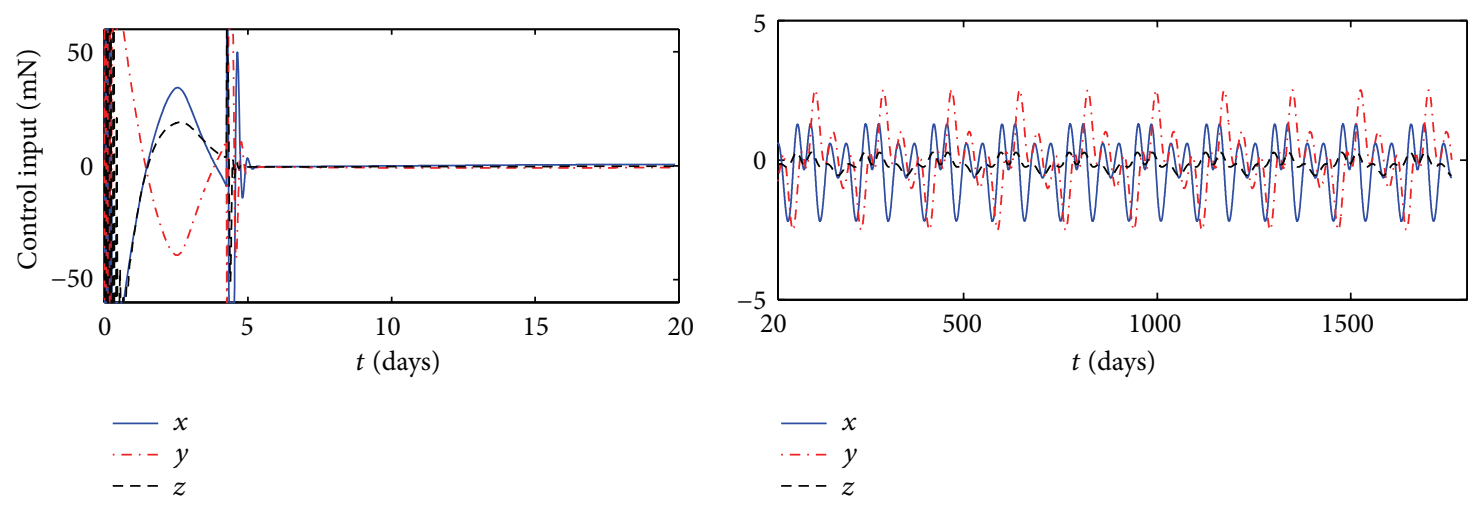

(c)

FIGURE 5: The relative position errors, velocity errors, and control input of ADRSC control of ISEE-3.

for station-keeping missions and do not have stringent orbittracking requirements. While high accuracy orbit stationkeeping is definitely required for current missions, which use continuous low-thrust propulsion.

\subsection{Halo Orbit $(Z=800,000 \mathrm{~km})$}

5.3.1. Related Parameters. The parameters of ADRSC of Halo orbit $(Z=800,000 \mathrm{~km})$ are the same as ADRSC of ISEE-3 mission except the parameters of self-turning $h_{i}: k=0.0120$, $h_{0}=-0.011$. Thus, the ADRSC can be easily taken into practice. The weight matrices of the LQR controller are selected as $Q=\operatorname{diag}\left\{4 \times 10^{8}, 4 \times 10^{8}, 4 \times 10^{8}, 10^{6}, 10^{6}, 10^{6}\right\}, R=\mathbf{I}_{3}$.

5.3.2. Simulation Results. Figures 7 and 8 illustrate the ADRSC results for Halo orbit $(Z=800,000 \mathrm{~km})$ with orbit injection errors as well as disturbing force. From Figures 8(a) and 8(b), one can find that ADRSC controller can drive the relative position errors and velocity errors along $x, y$, and $z$ steadily to zero within 7.5 days, compared with LQR controller 25 days in Figures 9(a) and 9(b). During the steady periodic, the position errors are kept within $2 \mathrm{~km}$ which illustrate high accurately maintained ability of ADRSC of high Halo orbit.

The velocity increment and the mean absolute value of the position errors of both ADRSC and LQR are presented in Table 3. The position error of ADRSC is $1.146 \mathrm{~km}$, which is greatly less than LQR controller $17.084 \mathrm{~km}$. Meanwhile, the control consumption of ADRSC is $195.248 \mathrm{~m} / \mathrm{s} / \mathrm{T}$, which is almost equal to that of LQR controller $195.394 \mathrm{~m} / \mathrm{s} / \mathrm{T}$.

5.4. Summary. From the above analyses and control results between ADRSC and LQR, we can find the following. 

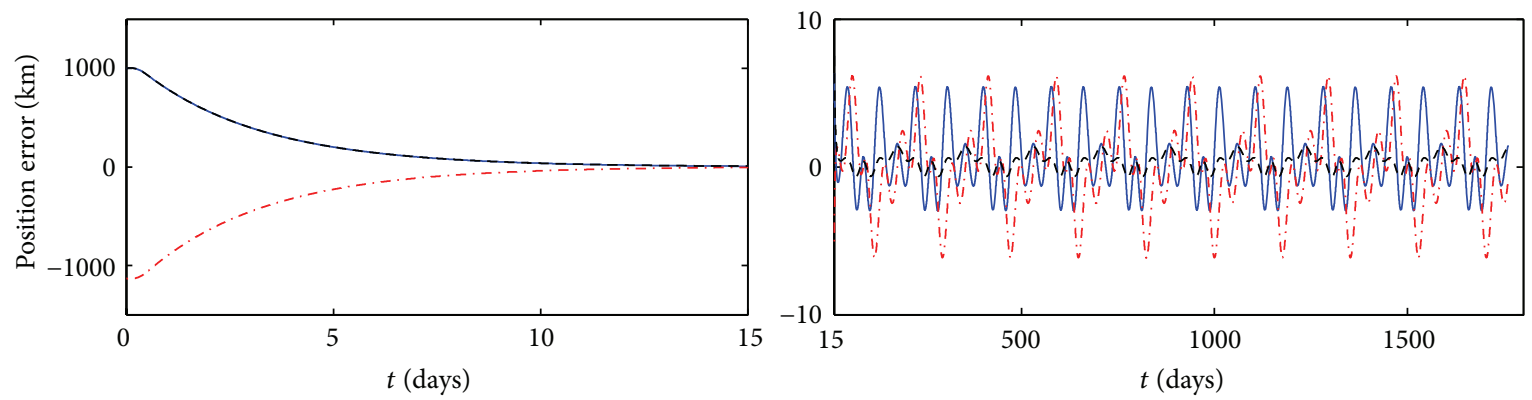

(a)
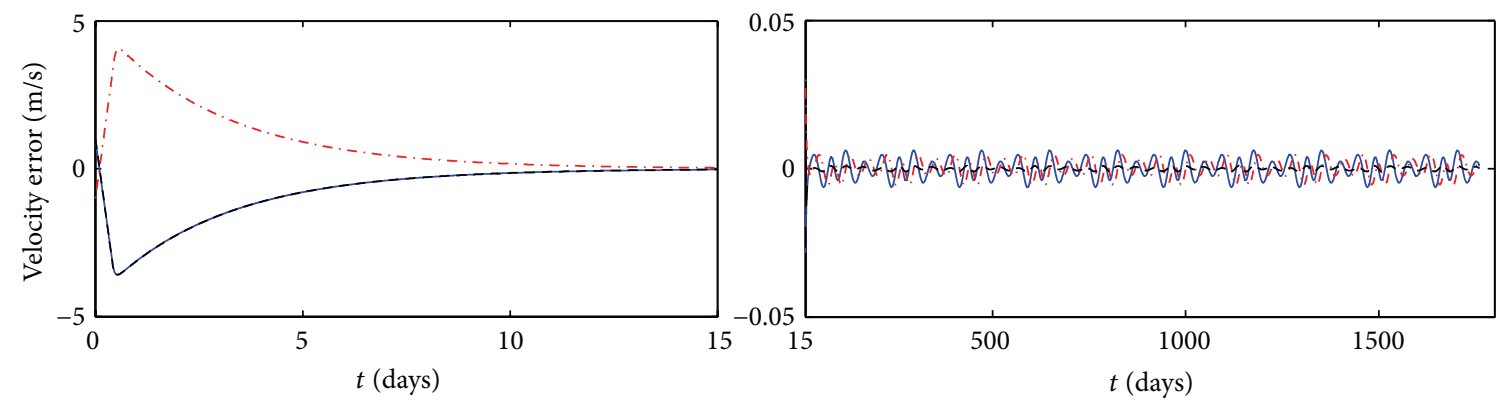

(b)
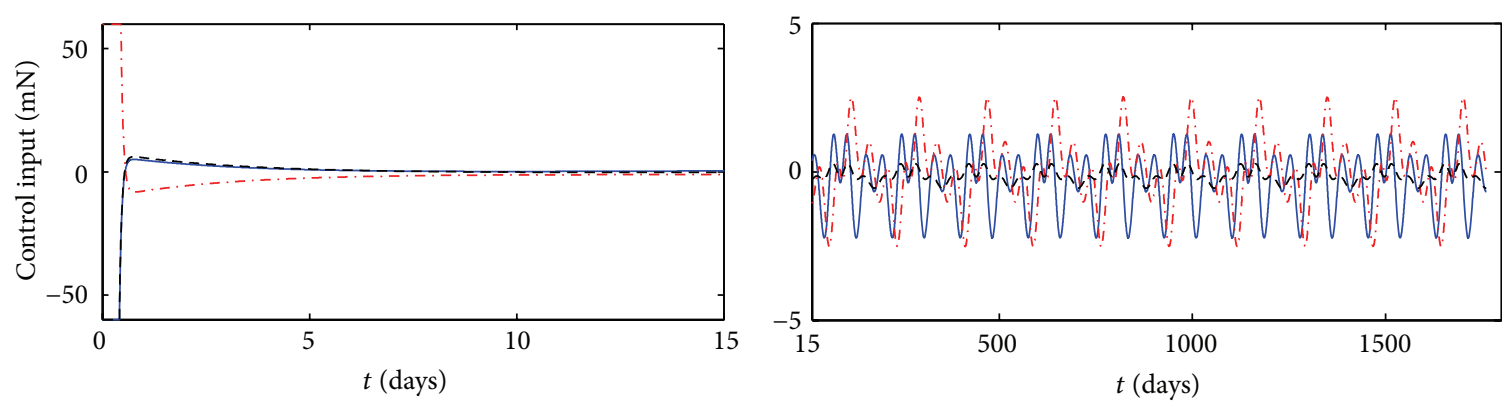

$$
\begin{aligned}
& -x \\
& -\cdots y \\
& --z
\end{aligned}
$$$$
\begin{aligned}
& -x \\
& -\cdot-y \\
& ---z
\end{aligned}
$$

(c)

FIGURE 6: The relative position errors, velocity errors, and control input of LQR control of ISEE-3.

\begin{tabular}{|c|c|c|c|c|c|c|c|c|}
\hline Mission orbits & $\Delta v_{x}$ & $\Delta v_{y}$ & $\Delta v_{z}$ & $\Delta v$ & $e_{x}$ & $e_{y}$ & $e_{z}$ & $e$ \\
\hline ADRSC: ISEE-3 $(110,000 \mathrm{~km})$ & 24.872 & 25.948 & 6.721 & 36.567 & 0.135 & 0.343 & 0.119 & 0.389 \\
\hline LQR: ISEE-3 (110,000 km) & 25.030 & 25.991 & 6.727 & 36.705 & 1.991 & 2.126 & 0.555 & 2.965 \\
\hline ADRSC: Halo $(800,000 \mathrm{~km})$ & 160.770 & 81.501 & 75.049 & 195.248 & 0.380 & 0.607 & 0.895 & 1.146 \\
\hline LQR: Halo $(800,000 \mathrm{~km})$ & 160.815 & 81.703 & 75.115 & 195.394 & 14.325 & 6.516 & 6.647 & 17.084 \\
\hline
\end{tabular}

TABLE 3: The velocity increments and the mean absolute value of the position errors of ADRSC and LQR control.

(1) The ADRSC, which is an error driven control method, is adequate for the station-keeping control of unstable orbits without any knowledge about the spacecraft dynamic model.

(2) The ADRSC presents praiseworthy station-keeping performance with orbit injection errors as well as unmodeled disturbances such as the SRP and the perturbative forces due to the eccentric nature of the Earth's orbit using an ionic engine with maximum thrust $60 \mathrm{mN}$.

(3) The ADRSC approach has better station-keeping control ability and higher orbit maintenance accuracy 


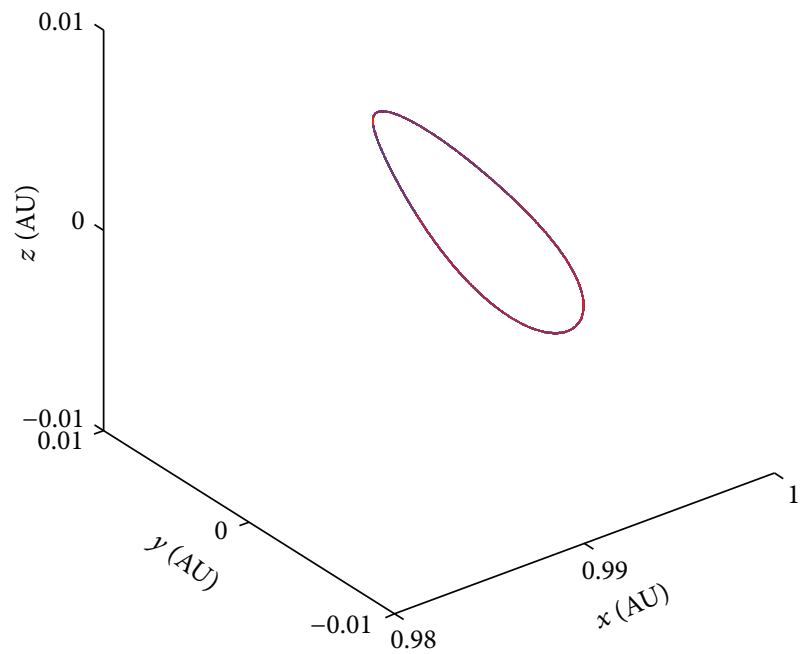

(a)

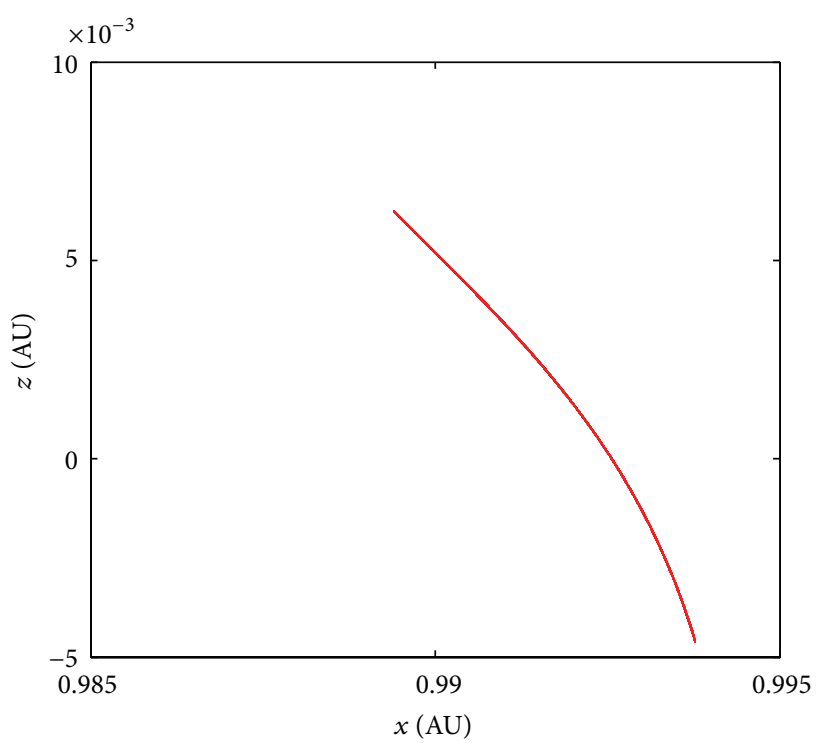

(c)

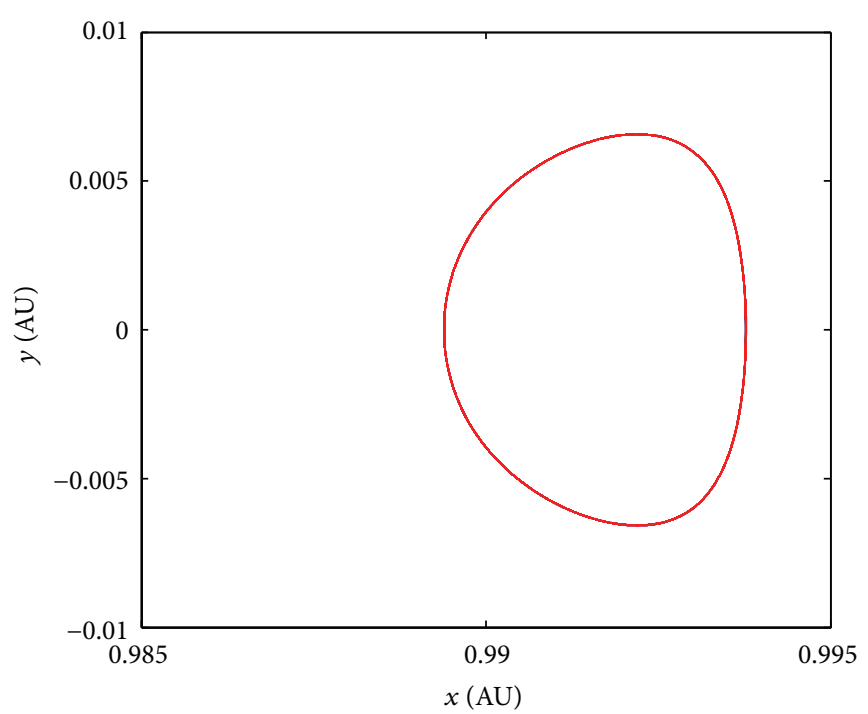

(b)

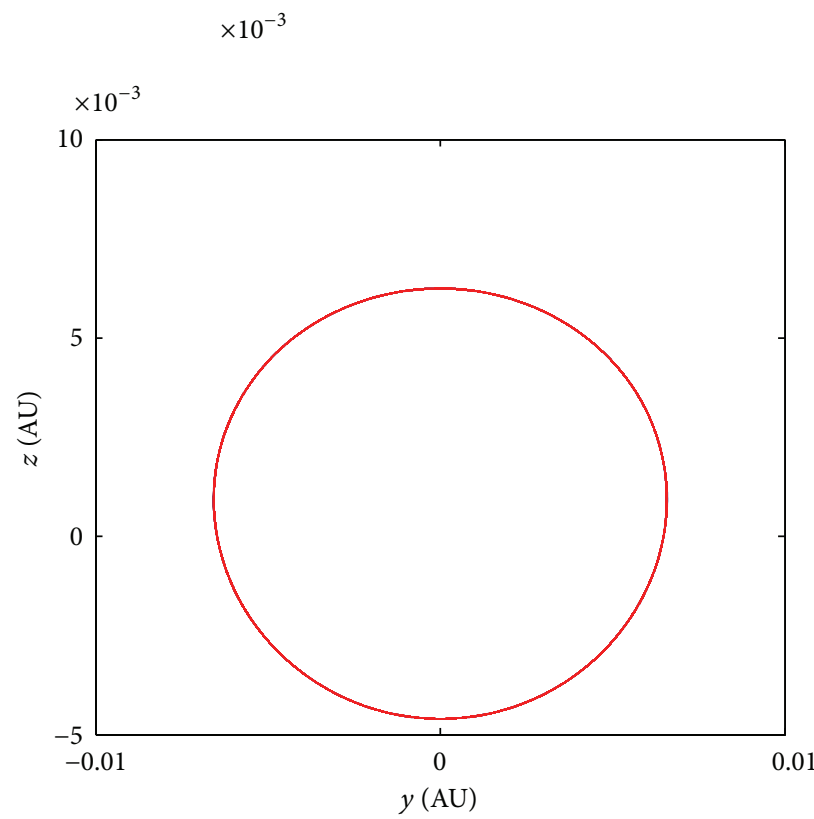

(d)

Figure 7: ADRSC control of Halo orbit ( $Z=800,000 \mathrm{~km})$.

compared with LQR controller despite of the same level of both the control methods thrust usage.

(4) The ADRSC has better robust performance compared with the general station-keeping method depending on spacecraft dynamic model.

There has been a great deal of commendable research on unstable libration point orbits station-keeping as mentioned before. A major point of departure between ADRSC method and earlier approaches to station-keeping is that ADRSC is an error driven rather than model-based control law, which can inherently get across the dependency on model accuracy as well as the drawbacks of linearization. With the combination of TD, ESO, and NLSEF, the unmodeled disturbances as well as the unmodeled system dynamic can be compensated in real time. Thus, fast response-time requirement and high accuracy of orbit maintenance requirement can be satisfied by ADRSC.

It is important to keep in mind not only the tracking accuracy but also the robustness of the station-keeping controller, as the space environment cannot be accurately modeled as well as the internal and external disturbance. ADRSC extends the unmodeled spacecraft dynamic and the disturbance as a state, which can be estimated from 

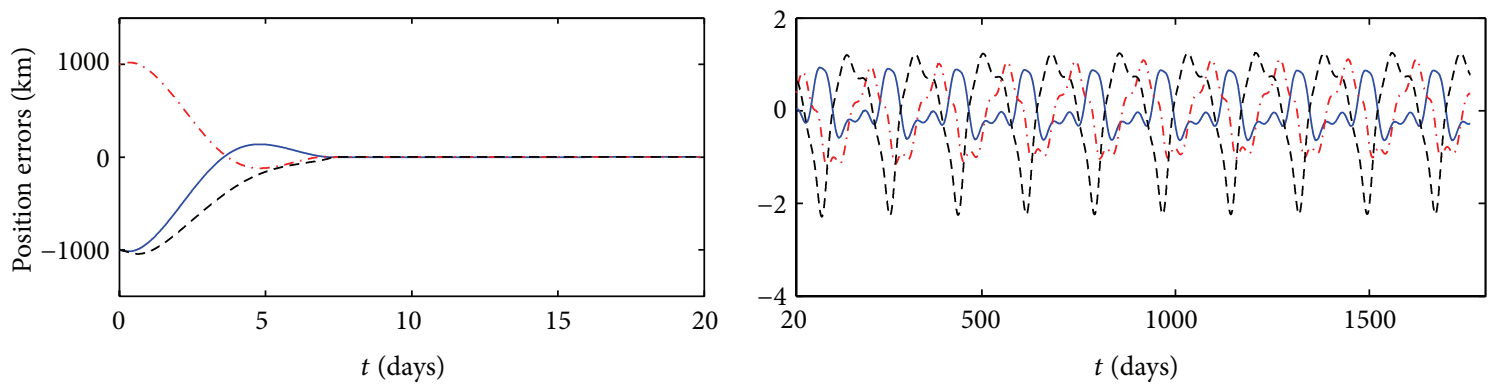

(a)
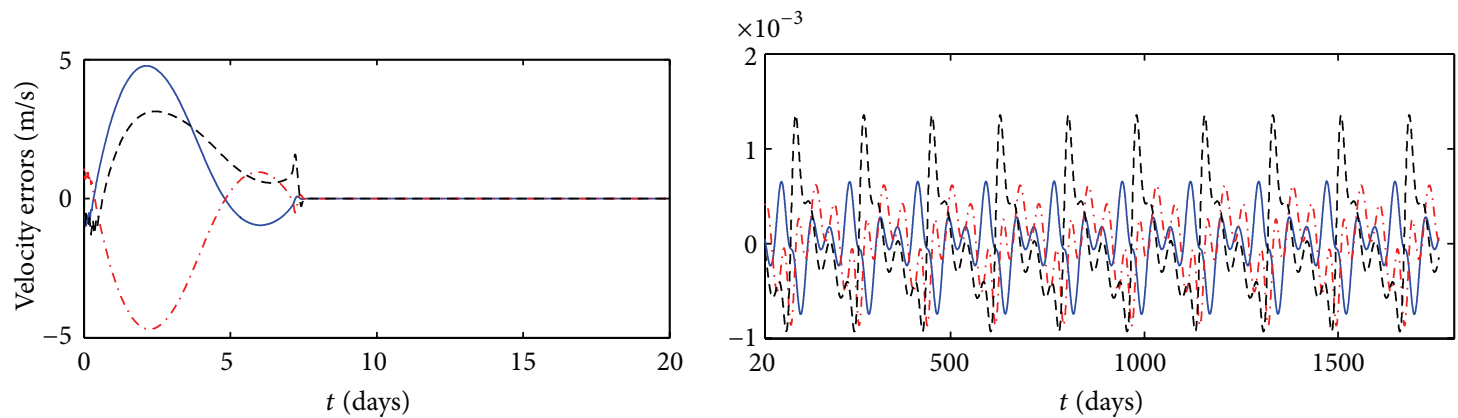

(b)
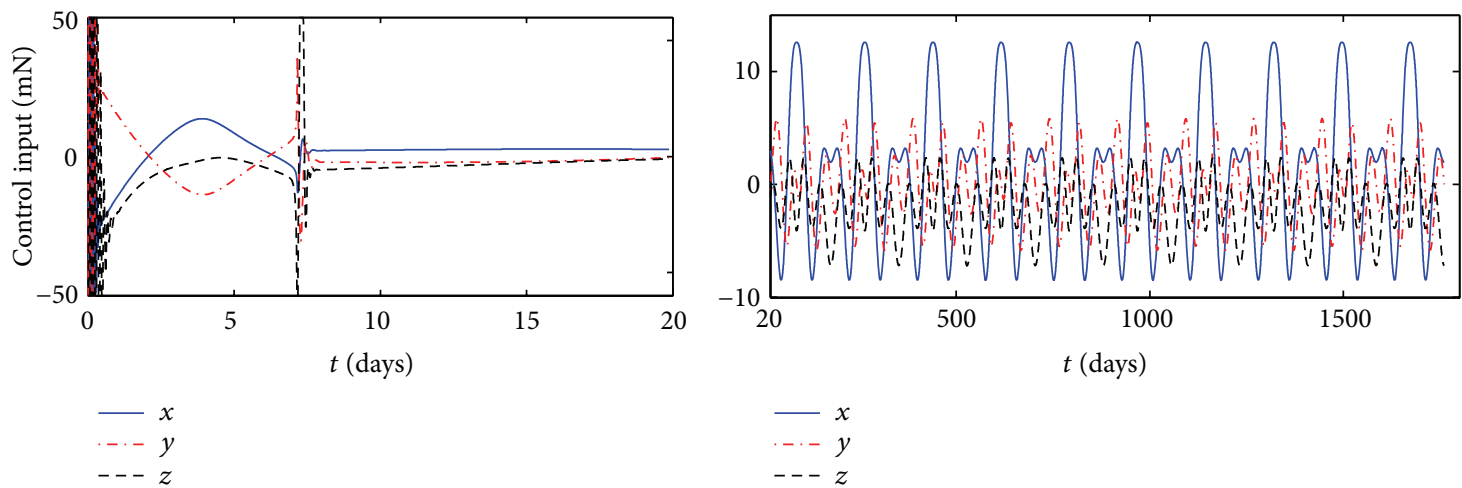

(c)

FIGURE 8: The relative position errors, velocity errors, and control input of ADRSC control of Halo orbit.

ESO and then "rejected" from nonlinear feedback control. Thus, no matter the spacecraft system is model known or unknown, linear or nonlinear, time invariant or time variant, with disturbance or without, ADRSC is able to show desired performance.

\section{Conclusion}

A successful ADRSC approach for station-keeping in the Sun-Earth $L_{1}$ point is accomplished using the input and output of nonlinear spacecraft dynamic system rather than precise nonlinear dynamic model or linearization. The nonlinear simulation results indicate that ADRSC is adequate for the station-keeping of unstable orbits that take into account the system uncertainties, initial injection errors, SRP, and perturbations of the eccentric nature of the Earth's orbit. With this ADRSC method, the system control performance is improved significantly by comparing with a general LQR method in the simulation. The thrust required by the control law is also reasonable and can be implemented using a continuous low-thrust propulsion device such as an ion engine. ADRSC can be qualified for future missions, which require better performance, more robustness, and higher station-keeping accuracy. It is anticipated that this method will work for other unstable orbits such as Lissajous and Halo orbits near other collinear points as well as the formation flying on these orbits which are the focus for future study. 

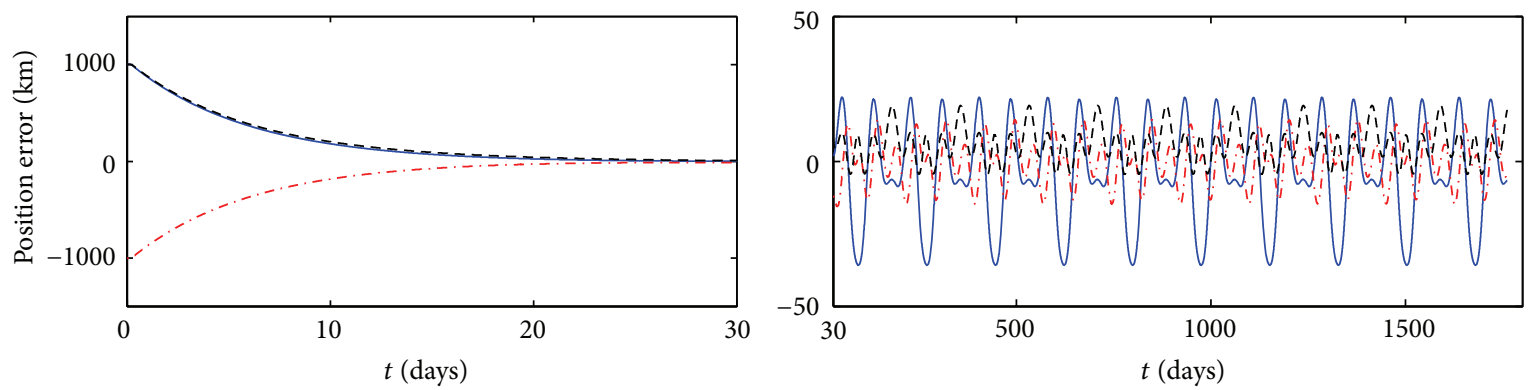

(a)
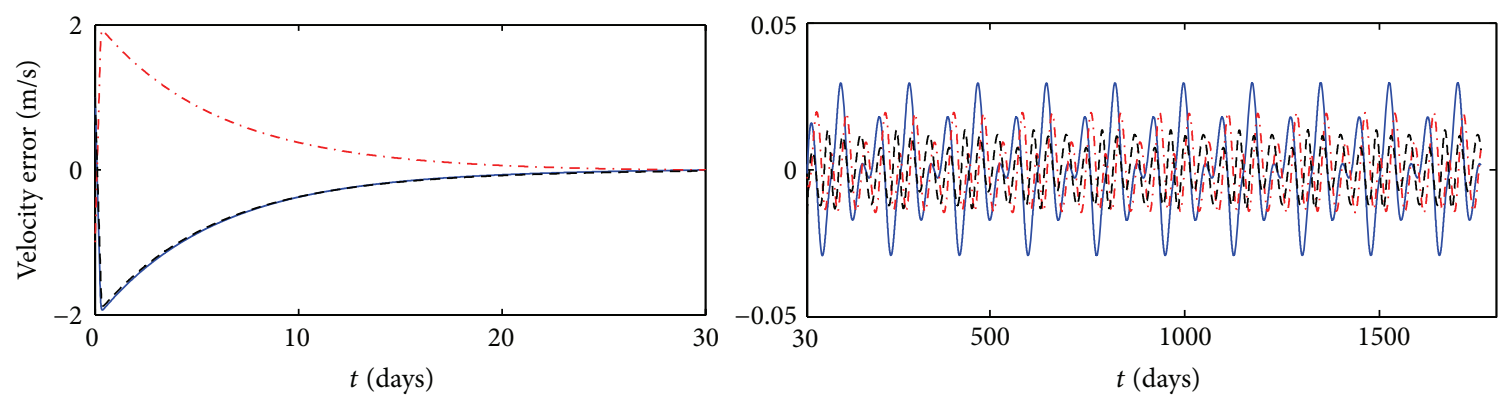

(b)
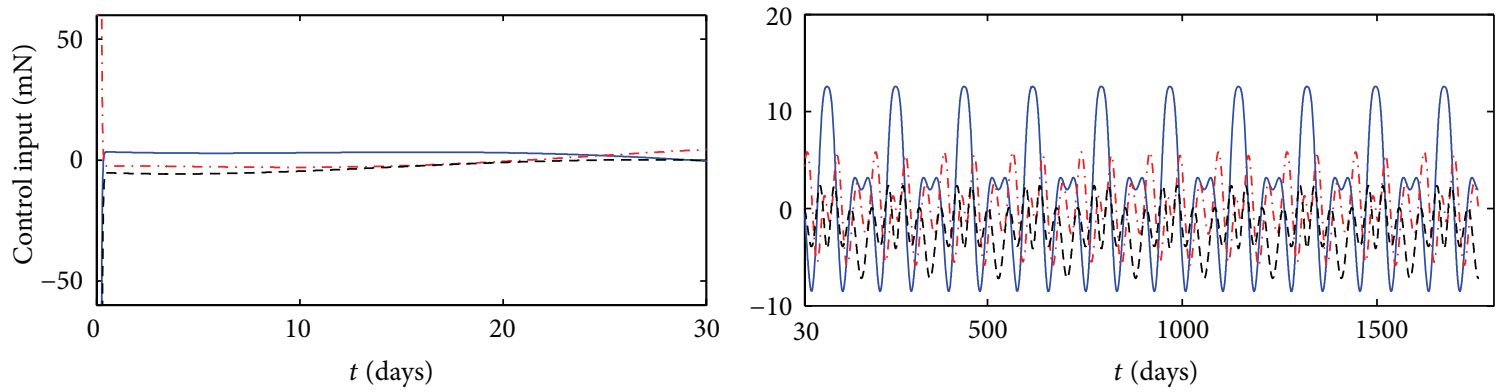

$$
\begin{aligned}
& -x \\
& -\cdot y \\
& --y
\end{aligned}
$$$$
\begin{aligned}
& -x \\
& -\cdot-y \\
& ---z
\end{aligned}
$$

(c)

FIGURE 9: The relative position errors, velocity errors, and control input of LQR control of Halo orbit.

\section{Conflict of Interests}

The authors declare that there is no conflict of interests regarding the publication of this paper.

\section{Acknowledgments}

This work is supported by National Nature Science Foundation of China under Grant no. 61004017 and by the PolishNorwegian Research Programme in the frame of Project Contract no. Pol-Nor/200957/47/2013. The authors would like to thank the editors and the anonymous reviewers for their keen and insightful comments which greatly improve the paper.

\section{References}

[1] B. Wie, Space Vehicle Dynamics and Control, AIAA, 1998.

[2] K. C. Howell, “Three-dimensional, periodic, halo orbits," Celestial Mechanics, vol. 32, no. 1, pp. 53-71, 1984.

[3] J. E. Kulkarni, M. E. Campbell, and G. E. Dullerud, "Stabilization of spacecraft flight in halo orbits: an $H_{\infty}$ approach," IEEE Transactions on Control Systems Technology, vol. 14, no. 3, pp. 572-578, 2006.

[4] M. Xin, S. N. Balakrishnan, and H. J. Pernicka, "Multiple spacecraft formation control with $\theta$-D method," IET Control Theory and Applications, vol. 1, no. 2, pp. 485-493, 2007.

[5] D. Sheng, X. Yang, and H. R. Karimi, "Robust control for autonomous spacecraft evacuation with model uncertainty and 
upper bound of performance with constraints," Mathematical Problems in Engineering, vol. 2013, Article ID 589381, 2013.

[6] F. Wang, X. Chen, A. Tsourdos, B. A. White, and X. Cao, "Sunearth $L_{2}$ point formation control using polynomial eigenstructure assignment," Acta Astronautica, vol. 76, no. 3, pp. 26-36, 2012.

[7] Z. Li, M. Liu, H. R. Karimi, and X. Cao, "Observer-based stabilization of spacecraft rendezvous with variable sampling and sensor nonlinearity," Mathematical Problems in Engineering, vol. 2013, Article ID 902452, 11 pages, 2013.

[8] Z. Li, M. Liu, H. R. Karimi, and X. Cao, "Sampled-data control of spacecraft rendezvous with discontinuous lyapunov approach," Mathematical Problems in Engineering, vol. 2013, Article ID 814271, 10 pages, 2013.

[9] J. V. Breakwell, "Investigation of halo satellite orbit control," Tech. Rep. CR-132 858, NASA, 1973.

[10] J. V. Breakwell, A. A. Kamel, and M. J. Ratner, "Station-keeping for a translunar communication station," Celestial Mechanics, vol. 10, no. 3, pp. 357-373, 1974.

[11] J. Erickson and A. Glass, "Implementation of ISEE-3 trajectory control," in American Astronautical Society and American Institute of Aeronautics and Astronautics, Astrodynamics Specialist Conference, Provincetown, UK, 1979.

[12] D. Cielaszyk and B. Wie, "New approach to halo orbit determination and control," Journal of Guidance, Control, and Dynamics, vol. 19, no. 2, pp. 266-273, 1996.

[13] P. Di Giamberardino and S. Monaco, "On halo orbits spacecraft stabilization," Acta Astronautica, vol. 38, no. 12, pp. 903-925, 1996.

[14] P. Gurfil and N. J. Kasdin, "Stability and control of spacecraft formation flying in trajectories of the restricted three-body problem," Acta Astronautica, vol. 54, no. 6, pp. 433-453, 2004.

[15] C. Simó, G. Gómez, J. Llibre, R. Martínez, and J. Rodríguez, "On the optimal station keeping control of halo orbits," Acta Astronautica, vol. 15, no. 6, pp. 391-397, 1987.

[16] G. G. Gómez, J. Llibre, R. Martínez, and C. Simó, Dynamics and Mission Design Near Libration Point Orbits, vol. 1 of Fundamentals: The Case of Collinear Libration Points, World Scientific, Singapore, 2001.

[17] G. G. Gómez, Dynamics and Mission Design Near Libration Points, vol. 3 of Advanced Methods for Collinear Points, World Scientific, 2001.

[18] K. C. Howell and H. J. Pernicka, "Stationkeeping method for libration point trajectories," Journal of Guidance, Control, and Dynamics, vol. 16, no. 1, pp. 151-159, 1993.

[19] A. Rahmani, M.-A. Jalali, and S. Pourtakdoust, "Optimal approach to halo orbit control," in Proceedings of the AIAA Guidance, Navigation, and Control Conference and Exhibit, pp. 11-14, Austin, Tex, USA, 2003.

[20] B. G. Marchand and K. C. Howell, "Control strategies for formation flight in the vicinity of the libration points," Journal of Guidance, Control, and Dynamics, vol. 28, no. 6, pp. 1210-1219, 2005.

[21] P. Gurfil, M. Idan, and N. J. Kasdin, "Adaptive neural control of deep-space formation flying," Journal of Guidance, Control, and Dynamics, vol. 26, no. 3, pp. 491-501, 2003.

[22] X. Bai and J. L. Junkins, "Modified Chebyshev-Picard iteration methods for station-keeping of translunar halo orbits," Mathematical Problems in Engineering, vol. 2012, Article ID 926158, 18 pages, 2012.
[23] J. Han, "Control theory, is it a model analysis or direct control approach," Journal of Systems Science and Mathematical Science, vol. 9, no. 4, pp. 328-335, 1989.

[24] J. Han, "From PID to active disturbance rejection control," IEEE Transactions on Industrial Electronics, vol. 56, no. 3, pp. 900906, 2009.

[25] D. Sun, "Comments on active disturbance rejection control," IEEE Transactions on Industrial Electronics, vol. 54, no. 6, pp. 3428-3429, 2007.

[26] G. E. Dullerud and F. Paganini, A Course in Robust Control Theory, vol. 6, Springer, New York, NY, USA, 2000.

[27] D. L. Richardson, "Halo orbit formulation for the isee-3 mission," Journal of Guidance, Control, and Dynamics, vol. 3, no. 6, pp. 543-548, 1980.

[28] D. L. Richardson, "Analytic construction of periodic orbits about the collinear points," Celestial Mechanics, vol. 22, no. 3, pp. 241-253, 1980.

[29] S. Yin, S. X. Ding, A. Haghani, H. Hao, and P. Zhang, "A comparison study of basic data-driven fault diagnosis and process monitoring methods on the benchmark tennessee eastman process," Journal of Process Control, vol. 22, no. 9, pp. 1567-1581, 2012.

[30] S. Yin, S. X. Ding, A. H. Abandan Sari, and H. Hao, "Data-driven monitoring for stochastic systems and its application on batch process," International Journal of Systems Science, vol. 44, no. 7, pp. 1366-1376, 2013.

[31] J. Han and W. Wang, "Nonlinear tracking-differentiator," Journal of Systems Science and Mathematical Science, vol. 14, no. 2, pp. 177-183, 1994.

[32] J. Han, "The "extended state observer" of a class of uncertain systems," Control and Decision, vol. 10, no. 1, pp. 85-88, 1995.

[33] H. Peng, J. Zhao, Z. Wu, and W. Zhong, "Optimal periodic controller for formation flying on libration point orbits," Acta Astronautica, vol. 69, no. 7-8, pp. 537-550, 2011.

[34] P. Gurfil, "Control-theoretic analysis of low-thrust orbital transfer using orbital elements," Journal of Guidance, Control, and Dynamics, vol. 26, no. 6, pp. 979-983, 2003.

[35] M. Martinez-Sanchez and J. E. Pollard, "Spacecraft electric propulsion-an overview," Journal of Propulsion and Power, vol. 14, no. 5, pp. 688-699, 1998. 


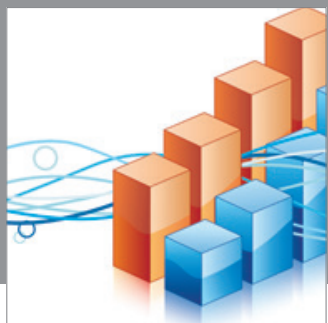

Advances in

Operations Research

mansans

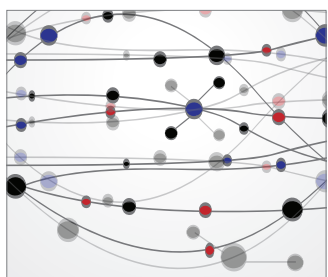

The Scientific World Journal
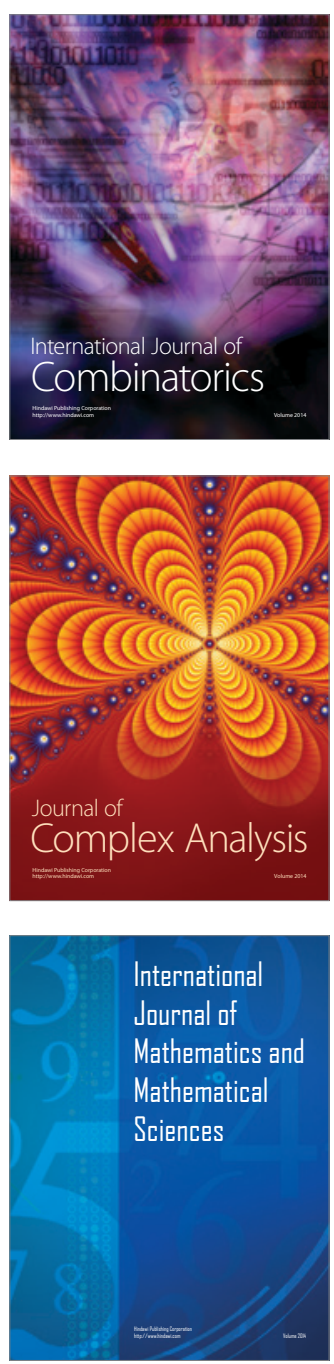
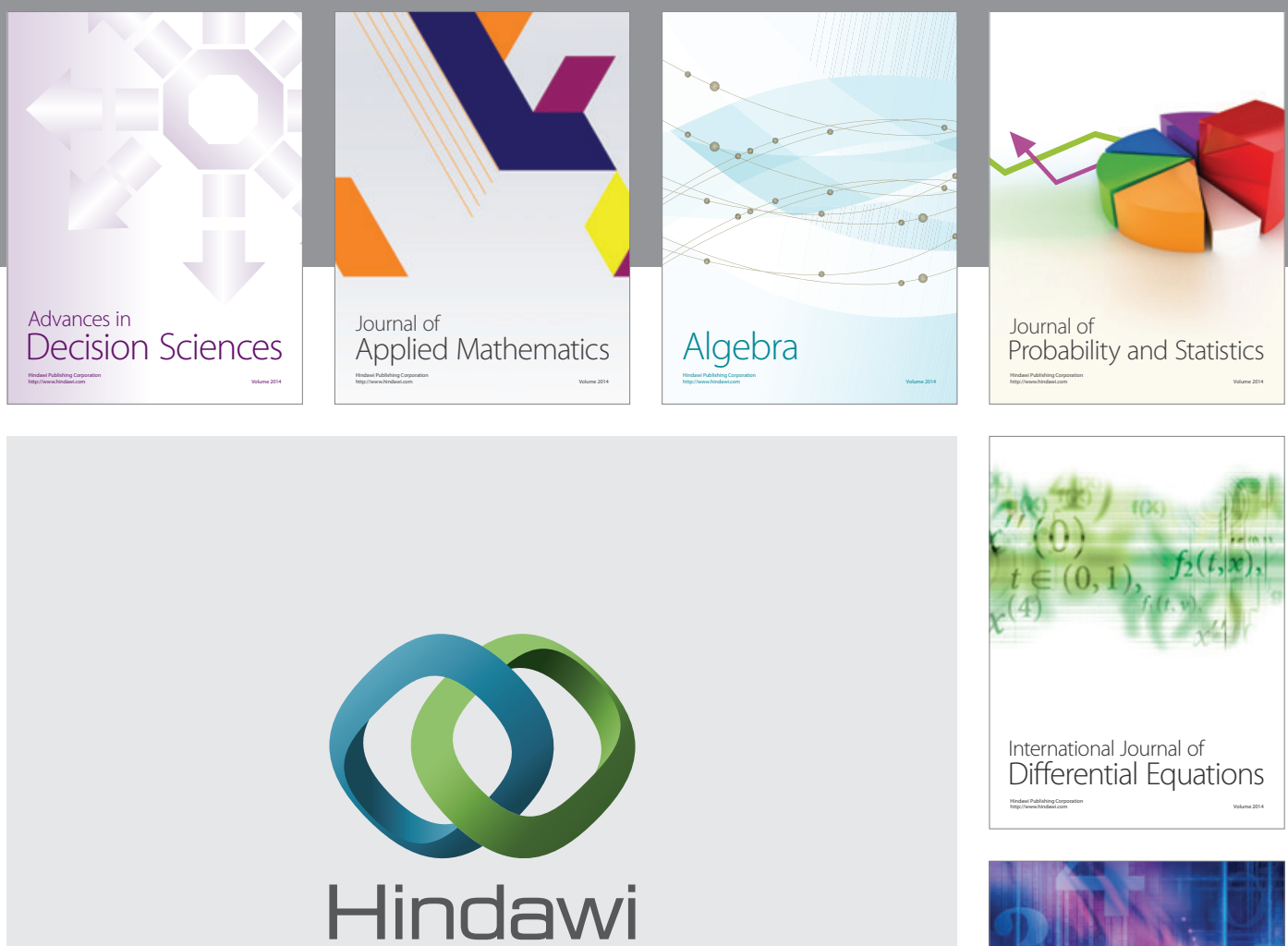

Submit your manuscripts at http://www.hindawi.com
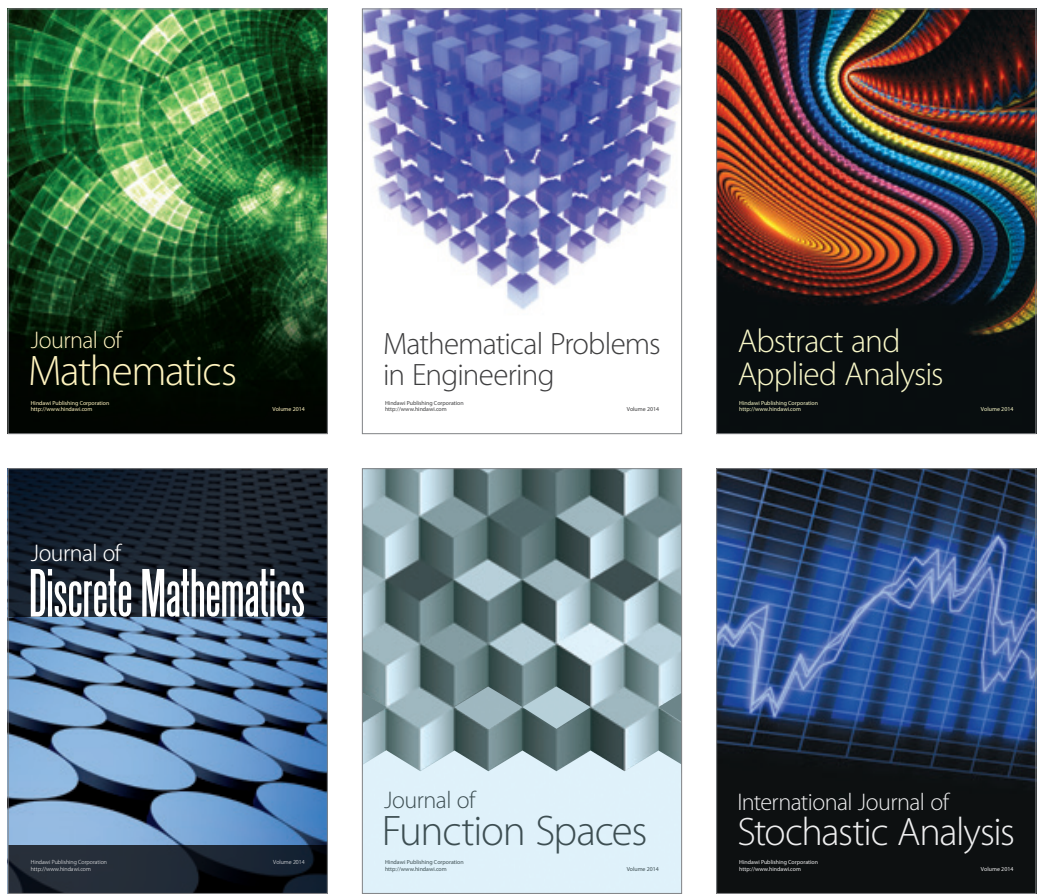

Journal of

Function Spaces

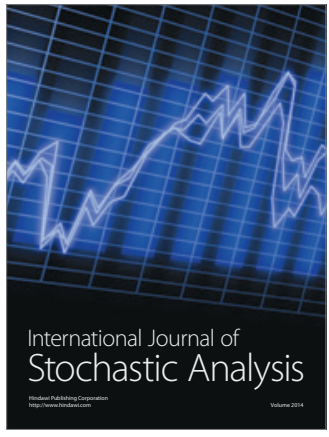

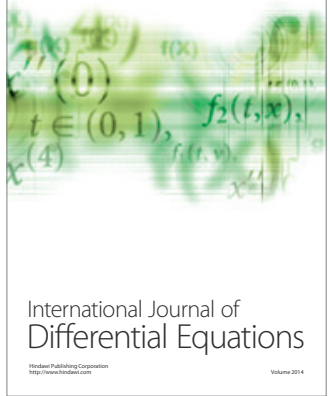
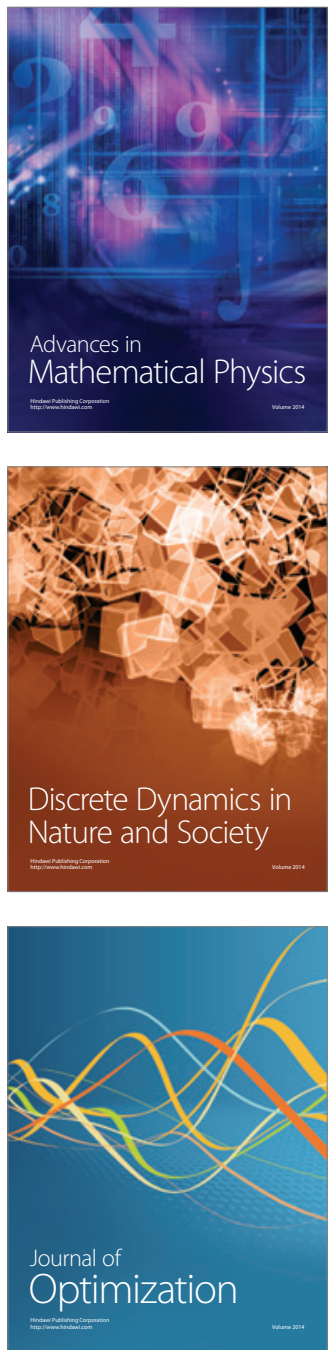